\title{
$\$$ Research Square

\section{Transcriptome analysis and differentially expressed gene screening for hypoxic-ischemic brain damage in rats treated with acupuncture}

\section{Xin Jiang}

Guangzhou University of Chinese Medicine

\section{Weixun Qin}

Shaanxi Provincial Hospital of Traditional Chinese Medicine

Junyan Wu

Guangzhou University of Chinese Medicine

Jiang Xiao

Guangzhou University of Chinese Medicine

Yue Zhong

Guangzhou University of Chinese Medicine

Chao Yuan

Guangzhou University of Chinese Medicine

Qing Yuan ( $\nabla$ yuanqing@gzucm.edu.cn )

Guangzhou University of Chinese Medicine

\section{Research Article}

Keywords: Hypoxic-ischemic brain damage (HIBD), long non-coding RNAs(IncRNA), critical diseases, Kyoto Encyclopedia of Genes and Genomes (KEGG), Gene Ontology (GO)

Posted Date: February 24th, 2021

DOI: https://doi.org/10.21203/rs.3.rs-228827/v1

License: (a) (1) This work is licensed under a Creative Commons Attribution 4.0 International License. Read Full License 


\section{Abstract}

Hypoxic-ischemic brain damage (HIBD) is one of the most common critical diseases in neonates with high mortality and disability rates. The latest research showed that long non-coding RNAs(IncRNA) played an important role in the development of HIBD. Recently, acupuncture therapy has been found to be effective in the treatment of HIBD. However, the mechanism of IncRNA in acupuncture treatment of HIBD is still unclear. In this study, we investigated the role of IncRNA in acupuncture treatment of HIBD in detail. We demonstrated behavioral performance similar to cognitive deficits in HIBD rat models in the new object recognition experiment and pathological lesion of the prefrontal cortex in nissl staining. Acupuncture treatments at acupoints DU24 and GB13 was proved to be effective in alleviating behavioral deficits and brain injury. A whole transcriptome analysis was applied to investigate transcriptome changes caused by acupuncture in PFC of HIBD rats. A total of 48 mRNAs and 65 IncRNAs was identified relate to acupuncture group and model group. According to Kyoto Encyclopedia of Genes and Genomes (KEGG) and Gene Ontology (GO) analysis, we found several IncRNAs and their target mRNAs were related to PI3K-Akt signaling pathway, TNF signaling pathway and NOD-like receptor signaling pathway, etc. The results of our research may provide new perspectives on the mechanism of acupuncture and affect the diagnosis and treatment of HIBD.

\section{Introduction}

Neonatal hypoxic-ischemic brain disorder (HIBD) is an important cause of neonatal death and birth defects 1 . Reportedly, the incidence of brain injury caused by perinatal asphyxia is $0.2 \%-0.4 \% 2$. What is worse, $20 \%-50 \%$ of the children with HIBD die in the neonatal period, and $>25 \%$ of the survivors develop permanent neurological disorders, such as mental retardation, cerebral palsy, epilepsy, and autism2,3. Presently, no international guidelines for the treatment of HIBD have been established, and the relatively common hypothermia therapy, stem cell transplantation, and melatonin pharmacological treatments 4,5 cannot fundamentally change the nerve defects and ischemic and hypoxic internal environment of children with HIBD. In China, acupuncture, as an alternative therapy, is widely used to treat various sequelae caused by HIBD, with significant clinical efficacy and no obvious side effects 6-9. Researchers have been constantly exploring the specific mechanisms of acupuncture treatment for HIBD 10-12. However, no definite conclusion has been reached on the target of acupuncture. Therefore, it is of great scientific significance to strengthen the research on the pathogenesis of neonatal ischemic and hypoxic encephalopathy and the therapeutic effect of acupuncture treatment.

Long non-coding RNA (IncRNA) is a type of non-coding RNA (ncRNA) with lengths ranging from $200 \mathrm{nt}$ to $100 \mathrm{~kb}$ that lack a significant open reading frame (ORF). Studies have shown that IncRNAs could regulate the potential molecular mechanisms of various biological processes, including chromatin tissue, epigenetic regulation, gene transcription, RNA conversion, and genome defenses 13-15. Additionally, IncRNA is involved in many important physiological functions of neurons in the nervous system playing an important role in the human brain development and the growth and evolution of higher cognitive functions 16. In recent years, a number of studies have realized the expression spectrum determination of 
IncRNAs in the brain tissue of HIBD-induced hypoxia and ischemia in neonatal rats through highthroughput sequencing technology, and some IncRNAs with significant changes have been identified. Ruibin Zhao et al 17 found that HIBD-induced brain injury changed the expression profile of IncRNA in newborn rats; for example, ENSRN OG00000021987 was down-regulated, and silencing of IT aggravated the apoptosis of cells in the hippocampus. $\mathrm{Li} \mathrm{H}$ et al 18 found that IncRNA TCONS_00044595 L was involved in the regulation of CLOCK in a post-transcriptional manner, which was likely to lead to a circadian rhythm disorder in newborns after HIBD. Fengyan Zhao et al 19 found that knocking out IncRNA BC088414 could reduce cell apoptosis and promote cell proliferation. However, there was no study on whether acupuncture can cause changes in the expression profile of IncRNAs in the prefrontal lobe of the brains of hypoxic-ischemic newborn rats caused by HIBD.

To investigate whether acupuncture can improve the cognitive ability of HIBD rats and reduce their brain injury, and to detail the gene expression level of RNA regulated by acupuncture, we conducted a new object recognition experiment and Nissl staining after acupuncture treatment at the acupoints DU24 and GB13 in HIBD rats. Additionally, we used RNA-sequencing (RNA-seq) technology to identify the changes in IncRNAs, as well as the mRNAs and circRNAs, in the prefrontal cortex (PFC) of HIBD newborn rats after treatment with acupuncture. Furthermore, the interaction network of differentiated miRNA-IncRNA or circRNA-mRNA was studied to find new pathways that participate in the mechanisms of neonatal HIBD. The results of this study may provide a theoretical basis for specific molecular biomarkers and targets of acupuncture therapy for neonatal HIBD.

\section{Results}

\section{Novel object recognition}

After acupuncture intervention, there was no significant difference in the exploration time for left object (TI) and exploration time for right object (Tr) of the rats in the normal, model, and acupuncture groups which indicated that the rats in each group didn't show preference for the location of the objects. The exploration time for familiar object (Tf) and exploration time for novel object (Tn) of the rats between the normal, model, and acupuncture groups in the test period were statistically significantly different. Furthermore, the difference in the relative discrimination index (DI) value between the model and normal groups was statistically significant $(P<0.001)$. The difference in the DI value between the acupuncture and model groups was also statistically significant $(P<0.001)$. The results of novel object recognition suggested that acupuncture could significantly improve the new object discrimination ability of the HIBD rats (Fig. 1).

\section{Nissl staining}

Nissl staining was used to observe the level of neuronal injury. There was no significant change in the thickness of the PFC in the normal, model, and acupuncture groups. However, compared with the normal 
group, the number of Nissl bodies was significantly decreased and disordered in the model group. The amount of Nissl bodies in the acupuncture group was also less than that in the normal group but was more than that in the model group (Fig. 2).

\section{RNA-seq of PFC}

To fully elucidate the mechanism of action of acupuncture on IncRNA and mRNA of HIBD rats, RNA-seq technology was applied to the acupuncture, model, and normal groups of young rats (four samples per group). We selected the PFC, which is a brain area with a high association of HIBD, to be the sampling part. After strict control of data quality, mapping, and assembling of transcripts, a total of $1,371,417,384$ clean reads were separated from the total reads of 1,304,758,138 raw reads. Then, we predicted the coding potential of IncRNA by CPC2, Pfam, and CNCl. Finally, a total of 15,525 IncRNAs were selected for further study.

\section{Identification and characteristic comparisons of IncRNAs and mRNAs}

A total of 174 differentially expressed mRNAs were identified in total. The results of the clustering analysis of differentially expressed mRNAs were illustrated with a heat map (Fig. 3A). According to the restrictively screened condition FDR $<0.05$ and $P<0.05,103$ mRNAs were significantly and differentially expressed in the model rats relative to the normal rats with 53 up-regulated and 53 down-regulated (Fig. 3B); 85 mRNAs were significantly and differentially expressed in acupuncture rats relative to model rats with 34 up-regulated and 51 down-regulated (Fig. 3C). Venn analysis showed that acupuncture treatment reversed 10 down-regulated mRNA (ENSRNOT00000021857, ENSRNOT00000071615, ENSRNOT00000088376, ENSRNOT00000080832, ENSRNOT00000081497, ENSRNOT00000089292, ENSRNOT00000089938, ENSRNOT00000088188, TCONS_00345929, ENSRNOT00000003297) and 7 upregulated mRNAs (ENSRNOT00000042539, ENSRNOT00000092470, ENSRNOT00000086014, ENSRNOT00000061858, TCONS_00150509, TCONS_00143419, ENSRNOT00000086397) of the model group (Fig. 3D-E).

There were 258 differentially expressed IncRNAs identified. The results of the clustering analysis of differentially expressed IncRNAs were illustrated with a heat map (Fig. 4A). Screening with the same method as above, 202 IncRNAs were significantly and differentially expressed in the model rats relative to the normal rats with 158 up-regulated and 44 down-regulated (Fig. 4B); 77 IncRNAs were significantly and differentially expressed in the rats that received acupuncture relative to the model rats with 31 upregulated and 46 down-regulated (Fig. 4C). As the Venn analysis showed, the 14 up-regulated IncRNAs (TCONS_00038506, TCONS_00105149, TCONS_00021584, TCONS_00216724, TCONS_00310736, TCONS_00216955, TCONS_00068680, TCONS_00163999, TCONS_00004602, TCONS_00013568, TCONS_00118348, TCONS_00025912, TCONS_00181752, TCONS_00092759) decreased in the 
acupuncture group, whereas 7 down-regulated IncRNAs (TCONS_00206366, TCONS_00097918, TCONS_00049434, TCONS_00364333,TCONS_00010371, TCONS_00250258,TCONS_00001104) increased in the acupuncture group (Fig. 4D-E). Table 1 lists the parts of the potential targets of IncRNAs that are common and were significantly differentially expressed. Moreover, we found that the distinct characteristic of IncRNAs and mRNAs was that IncRNAs had fewer exons, shorter lengths, and shorter lengths of the ORF than mRNAs (Fig. 5).

Table 1

IncRNA and potential target mRNAs

\begin{tabular}{|c|c|}
\hline IncRNAs & Potential target mRNAs \\
\hline TCONS_00013568 & Epo, Cdkn2a, Olr235, Wfdc16, LOC499584, Pdcd1lg2, Cmtm1, Vom1r87 Vgll2 \\
\hline TCONS_00068680 & $\begin{array}{l}\text { ॥9r, Csf3, Fshr, Upp2, Ly49i7, Fmr1nb, LOC100911801, rim52, Trpv5, Clrn3, } \\
\text { Apoa4 }\end{array}$ \\
\hline TCONS_00310736 & Th, Slc6a3, Htr1f, Trdn, Tnni1, Slurp2, Eomes, Gchfr, Alox15b \\
\hline TCONS_00163999 & $\begin{array}{l}\text { Ccl12, Car5a, Atp4b, Cd209f, Nuggc, LOC102547011, Fam24a, Elovl3, Xcr1, } \\
\text { Olr1694, Rhox2, Olr367, Tspo2 }\end{array}$ \\
\hline TCONS_00001104 & Gykl1, II24, Tcf24, RGD1565071, RGD1561671 \\
\hline TCONS_00173912 & $\begin{array}{l}\text { Reln, Galp, Ccl7, Shc4, Lmod2, Glt6d1, Irx4, Cabs1, Tex44, Sfrp5, Igll1, Olr1126, } \\
\text { Tfap2e }\end{array}$ \\
\hline TCONS_00057072 & Reln, Olr24, Pald1, Glyat12, Itpr3, AABR07004684.1, Acsm4, Igll1, Gpsm2 \\
\hline TCONS_00088551 & Reln, Olr299, Pgc, Afap1l2, Alx4, S1pr3, Gfral, Thsd7b, Ccl7 \\
\hline TCONS_00097914 & $\begin{array}{l}\text { Reln, Fcer1a, Itpr3, Col12a1, Gsap, Poteg, Saxo1, Shc4, Fev, Lmod2, Nid1, } \\
\text { Gpsm2, AC115384.1, Ell3, Tfap2e, Hoxc13 }\end{array}$ \\
\hline
\end{tabular}

CircRNA is a new type of RNA that comprises a covalently closed continuous loop structure with better structural stability than traditional linear RNA and could be highly expressed in eukaryotic transcriptome. The tissue and developmental specific expression were shown by circRNA, suggesting that they may play a key role in a variety of cellular processes. Remarkably, it has been reported that circRNAs could act as sponges of microRNA and participate in the regulation of genetic transcription.

There were 242 differentially expressed circRNAs identified. The results of the clustering analysis of differentially expressed circRNAs were illustrated with a heat map (Fig. 6A). At the same time, we found that 134 circRNAs were significantly and differentially expressed in the model rats relative to the normal rats with 59 up-regulated and 75 down-regulated (Fig. 6B); 127 circRNAs were significantly and differentially expressed in acupuncture rats relative to model rats with 59 up-regulated and 68 downregulated (Fig. 6C). As the Venn diagram suggested, 12 up-regulated circRNAs (novel_circ_0000993, novel_circ_0004188, novel_circ_0002854, novel_circ_0003148, novel_circ_0004103, novel_circ_0004184, novel_circ_0007805, novel_circ_0008253, novel_circ_0008750, novel_circ_0009942, novel_circ_0010967, and novel_circ_0014098) in the model group decreased in the acupuncture group, whereas seven down- 
regulated circRNAs (novel_circ_0013968, novel_circ_0000912, novel_circ_0002607, novel_circ_0005240, novel_circ_0008312, novel_circ_0011232, and novel_circ_0012046) in model group reversed in the acupuncture group (Fig. 6D-E).

\section{Bioinformatics}

To evaluate the potential cellular function of overlapped mRNAs, IncRNAs, and circRNAs between experimental groups, we conducted $\mathrm{GO}$ enrichment analysis across three domains, including molecular functions (MF), cellular components (CC), and biological processes (BP).

\section{Enrichment analysis: GO and KEGG of mRNAs}

Through the GO survey between the acupuncture and model groups, 1,522 GO terms were respectively enriched with $36 \mathrm{GO}$ terms having distinct statistical significance $(P<0.05$; Fig. 7A), with 29 in MF, 25 in $\mathrm{CC}$, and 236 in BP. The mRNAs that were differentially expressed between the acupuncture and model groups were involved in the positive regulation of glutamate metabolic processes, regulation of interleukin-2 production, histone H3-R17 methylation, and intracellular cAMP activated cation channel activity. In the GO enrichment analysis of the model and normal groups, 2,870 GO terms were respectively enriched with $52 \mathrm{GO}$ terms having distinct statistical significance $(P<0.05)$, with 24 in MF, 11 in CC, and 17 in BP. The mRNAs that were differentially expressed between the

model and normal groups were involved in the oxidation-reduction process, regulation of nuclease activity, proton-transporting ATP synthase complex, oxidoreductase activity, and transmembrane transporter activity (Fig. 7B).

We used the KEGG database for pathway analysis. The results showed that mRNAs that were differentially expressed between the acupuncture and model groups participated in multiple pathways, including morphine addiction, cholinergic synapses, primary bile acid biosynthesis, and glycosaminoglycan biosynthesis-heparan sulfate/heparin. In the comparison between the model and normal groups, the differentially expressed IncRNAs were related to chemical carcinogenesis, phototransduction, steroid hormone biosynthesis, and retinol metabolism. Figure 7C-D shows the most prominent pathways.

\section{Enrichment analysis: GO and KEGG of IncRNAs}

To evaluate the potential function of IncRNAs, we searched the target mRNAs of IncRNAs in cis and trans. Through the GO survey between the acupuncture and model groups, 4,619 GO terms were respectively enriched with $36 \mathrm{GO}$ terms having distinct statistical significance $(P<0.05)$, with 8 in MF, 8 in CC, and 20 in BP. The IncRNAs that were differentially expressed between the acupuncture and model groups were involved in neurological system processes, the plasma membrane, olfactory receptor activity, and 
receptor activity (Fig. 8A). In the GO enrichment analysis of the model and normal groups, 6,210 GO terms were respectively enriched with $37 \mathrm{GO}$ terms having distinct statistical significance $(P<0.05)$, with 10 in MF, 7 in CC, and 20 in BP. The IncRNAs differentially expressed between the model and normal groups were involved in olfactory receptor activity, G-protein-coupled receptor activity, and sensory perception (Fig. 8B).

We used the KEGG database for pathway analysis. The results showed that IncRNAs that were differentially expressed between the acupuncture and model groups participated in multiple pathways, including olfactory transduction, ribosomes, cytokine-cytokine receptor interactions, and taste transduction. In the comparison between the model and normal groups, the differentially expressed IncRNAs were related to pathways, such as olfactory transduction pathway, ribosomes, drug metabolism and other enzymes, and biosynthesis of amino acids. Figure 8C-D shows the most prominent pathways.

\section{Enrichment analysis: GO and KEGG analysis of circRNAs}

Through the GO survey, we found 2,145 GO terms that were respectively enriched between the acupuncture and model groups, whereas 2,613 GO terms were respectively enriched between the model and normal groups (Figure 9A-B). However, no GO term showed a significant statistical difference.

The results of the KEGG pathway analysis showed that circRNAs that were differentially expressed between the acupuncture and model groups participated in multiple pathways, including axon guidance, Wnt signaling pathway, B cell receptor signaling pathway, and GABAergic synapse. In the comparison between the model and normal groups, the differentially expressed IncRNAs may be related to pathways, such as adrenergic signaling in cardiomyocyte pathway, mTOR signaling pathway, neurotrophin signaling pathway, and MAPK signaling pathway. However, no pathway showed a significant statistical difference. Figure $9 \mathrm{C}-\mathrm{D}$ shows the most prominent pathways.

\section{miRNA-IncRNA/circRNA-mRNA interaction network}

MicroRNAs (miRNAs) are small endogenous RNAs that comprise 19 to 25 nucleotides and can regulate gene expression post-transcriptionally. Since the spongy peculiarity of miRNA, both IncRNA and mRNA can regulate miRNA function through the competing endogenous RNA (ceRNA) network In this study, differentially expressed mRNAs, IncRNAs, circRNAs and miRNAs between model and acupuncture groups were systematically identified by the whole transcriptome sequencing (RNA-seq). 30 circRNAs, 47 IncRNAs, and 11 mRNAs with differential expression were found that shared the same binding site for miRNA. In the same way, we found 30 mRNAs, 162 IncRNAs and 88 circRNAs with differential expression were on the common binding site of miRNA between the model and normal groups (Fig. 10).

\section{Discussion}


HIBD is a common disease found in newborns which often presents as a series of neurological sequelae. Acupuncture, as a therapy of alternative medicine, has achieved a remarkable curative effect in the treatment of this disease ${ }^{20,21}$. Meanwhile, several studies have documented the important neuroprotective roles of acupuncture in cerebral functional compensation in animal models with HIBD $22-$ ${ }^{24}$. With the rise of RNA-seq technology, people gradually became interested in the gene-related mechanism of HIBD and began to do research ${ }^{25,26}$. However, the role of acupuncture in regulating RNAs in HIBD rats remains unknown.

In this study, HIBD rats showed significantly longer new object recognition times, suggesting that HIBD rats may be worse than normal rats in terms of learning and memory. This result is consistent with a number of studies indicated that HIBD rats perform poorly in behavioral tests of learning and memory abilities, such as water maze, shuttle box, $T$ maze, and radial maze ${ }^{27-29}$. As a common neonatal nervous system disease, HIBD can lead to different degrees of brain damage, such as brain metabolism disorder, neuronal apoptosis, reduced brain flow, or even cerebral hemorrhage in severe cases, resulting in liquefaction and necrosis of brain tissue ${ }^{30-32}$. Our results were in line with these findings in that we found that there were significantly fewer Nissl corpuscles in the prefrontal lobe of HIBD rats, indicating that HIBD can indeed cause damage to neurons in the brains of rats. Acupuncture, as an alternative therapy, is attracting increasing attention for its ideal curative effect. Recently, it has been reported that acupuncture can improve locomotor activity and learning-memory ability by improving hippocampal cellular autophagy in rats with HIBD, suggesting that acupuncture could effectively alleviate the brain injury caused by HIBD ${ }^{33}$. Additionally, Yuan $\mathrm{Q}$ et al. found that acupuncture could significantly reduce the expression of CYT-C and caspase-3 in the cerebral cortex of HIBD rats and reduce the apoptosis of neurons to protect the brain tissue ${ }^{11}$. Consistent with these results, we found that the number of Neisseria bodies in HIBD rats treated with acupuncture was significantly higher than that in untreated rats. Additionally, the performance of the new object recognition experiment of the rats after acupuncture was better than that of the untreated HIBD rats, indicating that acupuncture is an effective therapy for HIBD.

In order to explore the target of acupuncture, we identified RNA differentially expressed in the PFC of three groups of rats and found something interesting: 1) IncRNAs had fewer exons, shorter lengths, and shorter lengths of the ORF than mRNAs; 2) from the gene heat map, we found that the effect of acupuncture intervention on IncRNA was more obvious than that of mRNA and circRNA. Moreover, the Venn diagram showed that the number of genes in the overlap part of IncRNA was higher than that of mRNA and circRNA; 3) 877 differentially expressed IncRNAs were identified, 65 IncRNAs were significantly and differentially expressed in acupuncture rats relative to model rats with 17 up-regulated and 48 downregulated, and 410 IncRNAs were significantly and differentially expressed in the model rats relative to the normal rats with 372 up-regulated and 38 down-regulated; 4) the GO analysis showed that IncRNAs that were differentially expressed between the acupuncture and model groups were involved in neurological system processes, plasma membrane, olfactory receptor activity, and receptor activity, whereas the IncRNAs that were differentially expressed between the model and normal groups were involved in 
olfactory receptor activity, G-protein-coupled receptor activity, and sensory perception. The KEGG pathway analysis revealed that IncRNAs that were differentially expressed between the acupuncture and model groups participated in multiple pathways, including olfactory transduction and ribosomes, cytokinecytokine receptor interactions, and taste transduction. In the comparison between the model and normal groups, the differentially expressed IncRNAs were associated with pathways, such as olfactory transduction, ribosomes, drug metabolism and other enzymes, and biosynthesis of amino acids; and 5) 30 circRNAs, 47 IncRNAs, and 11 mRNAs with differential expression shared a common binding site for miRNA between the acupuncture and model groups. Eighty-eight circRNAs, 162 IncRNAs, and 30 mRNAs with differential expression shared a common binding site for miRNA between the model and normal groups.

TCONS_00013568 was an up-regulated IncRNA in the model group but was decreased in the acupuncture group. Through KEGG pathway analysis, we found that the target gene of TCONS_00013568, Epo, was related to the Jak-STAT signaling pathway, HIF-1 signaling pathway, and PI3K-Akt signaling pathway. Reportedly, the inhibition of the JAK/STAT pathway can not only promote brain-derived neurotrophic factors and hippocampal neuron proliferation to alleviate autism symptoms ${ }^{34}$ but also protect against asynuclein-induced neuroinflammation and dopaminergic neurodegeneration ${ }^{35}$. Some researchers have found that the improvement in EPO was associated with the neuroprotective effect of the JAK/STAT pathway in cerebral palsy ${ }^{36}$. The HIF-1 signaling pathway was found to be associated with stroke, vascular dementia, and chronic hypoxia ${ }^{37-39}$. Activating the HIF-1 signaling pathway could exert angiogenic and anti-apoptotic effects against cerebral ischemia-reperfusion injury ${ }^{40}$. Hippocampal neurons could be protected against excessive autophagy and apoptosis through the PI3K/Akt signaling pathway ${ }^{41}$. EPO was found to promote cerebrovascular regeneration by activating the PI3K/Akt signaling pathway in premature brain damage ${ }^{42}$. In our results, Cdkn2a, the other target gene of TCONS_00013568, was also related to glioma and the p53 signaling pathway. The results of several studies showed that the p53 signaling pathway was closely related to Alzheimer's disease, glioblastoma multiforme, and ischemic stroke ${ }^{43-45}$. It has been reported that Cdkn2a plays an important role in apoptosis by activating the p53 signaling pathway ${ }^{46}$. The new target genes of TCONS_00013568 in the above pathways may provide new therapeutic targets for the treatment of HIBD. However, the detailed mechanism needs further study.

TCONS_00068680 was another up-regulated IncRNA in the model group with down-regulation in the acupuncture group. Several target genes of TCONS_00068680 participated in the Jak-STAT signaling pathway, such as $\| 9 r$ and Csf3. The expressed protein of $\| 9 r$ is the receptor of interleukin (IL)-9. Some researchers found that IL-9 was important in driving immune responses to chronic inflammation and that the blockade of IL-9 signaling in the Jak-STAT signaling pathway may be novel therapeutic targets for inflammatory diseases in the mucosal immune system ${ }^{47}$. Furthermore, IL-9 is closely associated with multiple sclerosis and could reduce inflammation and provide protection from neurodegeneration ${ }^{48}$. The expressed protein of Csf3 is a potent activator of neutrophil ROS, which is related to stroke. It was found to be neuroprotective in acute and chronic settings through various mechanisms, including the 
enhancement of neurogenesis and angiogenesis, as well as the apoptosis and suppression of inflammation ${ }^{49}$. Moreover, Csf3 was involved in the PI3K-Akt signaling pathway. However, there are few studies on Csf3 in the PI3K-Akt signaling pathway, and the mechanism still needs further study. Fshr was another target gene of TCONS_00068680, which was associated with the p53 signaling pathway. Fshr was reported to be related to apoptosis and cell proliferation ${ }^{50}$, but few studies investigated the mechanism of Fshr in the p53 signaling pathway in HIBD.

TCONS_00310736 was a IncRNA found to be up-regulated in the model group and down-regulated in the acupuncture group. Its target mRNAs, Th and Slc6a3, were related to dopaminergic synapses and Parkinson's disease. Dopaminergic synapses were found to be closely related to schizophrenia and Parkinson's disease ${ }^{51,52}$. The expressed protein of Th is the tyrosine hydroxylase, which was reported to inhibit the synthesis of dopamine and cause Parkinson's symptoms by decreasing its enzymatic activity 53. Moreover, the inhibition of Th in the substantia nigra could decrease the movement frequency 54 . Reportedly, Slc6a3 is strongly linked to dopamine transport. Hyperactivity, reduced sensitivity to reward, and impaired social behavior were measured in mutant rats with Slc6a3 knock-out ${ }^{55}$. Htr1f was another target mRNA of TCONS_00310736 and was found to participate in the p53 signaling pathway. However, there are few studies on Htr1f in the p53 signaling pathway, and the mechanism still needs further study.

TCONS_00163999 with its target mRNA, Ccl12, associated with the TNF signaling pathway and NOD-like receptor signaling pathway, was also a IncRNA that was up-regulated in the model group and downregulated in the acupuncture group. TNF plays an important role in nerve inflammation, and the TNF signaling pathway is closely related to ischemic stroke, Alzheimer's disease, Parkinson's disease, amyotrophic lateral sclerosis, and multiple sclerosis ${ }^{56}$. Reportedly, the functions of NOD-like receptors could be divided into four parts, namely, the inflammasome assembly, signaling transduction, transcription activation, and autophagy ${ }^{57}$. Moreover, the NOD-like receptor protein 3 (NLRP3) inflammasome has been found to be closely associated with proptosis in encephalomyelitis ${ }^{58}$. However, the mechanism of Ccl12 in the TNF signaling pathway and NOD-like receptor signaling pathway is not clear.

TCONS_00001104 was IncRNA down-regulated in the model group and up-regulated in the acupuncture group. Its target gene, Gykl1, is involved in the PPAR signaling pathway. Reportedly, activating the PPAR signaling pathway could significantly attenuate HI-induced brain injury by reducing neuronal apoptosis 59 . However, there are a few studies on the function of Gykl1 in the PPAR signaling pathway. II24, the other target gene of TCONS_00001104, was associated with the Jak-STAT signaling pathway. The expressed protein of IL-24 is interleukin-24, which was found to be closely related to apoptosis ${ }^{60}$.

Additionally, we found that two IncRNAs (TCONS_00173912 and TCONS_00057072) up-regulated in the model group and two IncRNAs (TCONS_00088551 and TCONS_00097914) down-regulated in the acupuncture group jointly regulate a target gene, Reln. Interestingly, Reln was found to be up-regulated in the model group and down-regulated in the acupuncture group and was closely related to the PI3K-Akt 
signaling pathway. Reln is reported to play an important role in neuronal structure maintenance of mature neurons and can regulate neuronal migration and synaptogenesis ${ }^{61,62}$. Moreover, some researchers found that Reln deficiency may be involved in the development of remote cognitive impairments and epigenetic regulation of DNA demethylation and histone acetylation of Reln might underlie the mechanisms of synaptic plasticity and memory retention in the medial PFC ${ }^{63,64}$. Reln also has a role in PI3-kinase signaling in neuronal growth cones, and it contributes to final neuron positioning in the mammalian brain by local modulation of protein kinase B and glycogen synthase kinase 3beta kinase activities ${ }^{65}$. The results of our study suggested that the increasing expression of TCONS_00173912 and TCONS_00057072 and the decreasing expression of TCONS_00088551 and TCONS_00097914 may inhibit Reln, which then works via the PI3K-Akt signaling pathway in neonatal HIBD.

In summary, we found several significant IncRNAs whose corresponding target genes might be the targets of acupuncture therapy for HIBD. At the same time, we investigated the miRNA-mRNA-IncRNA interaction network of the normal, HIBD, and acupuncture groups using RNA-seq analysis. To the best of our knowledge, this is the first research to study the expression of IncRNAs, circRNAs, and mRNAs between HIBD and acupuncture. The findings further expanded our understanding of ceRNA networks and will help us explore the functions of acupuncture. These novel networks may be potential biomarkers or therapeutic targets of acupuncture in HIBD. The research provides new perspectives on the mechanism of acupuncture and may affect the diagnosis and therapy of HIBD. However, this study also has its limitations because of its small sample size. In future studies, we will further expand the sample size and introduce clinical samples to provide a more sufficient basis for acupuncture treatment of HIBD.

\section{Methods}

\section{Animals}

Healthy pregnant specific pathogen-free Sprague-Dawley rats [SCXK (Guangdong) 2018-0002, No.44007200060611], weighing 350-450 g, were purchased from Guangdong Medical Laboratory Animal Center (Foshan, China) and then were kept under $12 \mathrm{~h}$ light/dark cycles at temperatures of $20^{\circ} \mathrm{C}-$ $26^{\circ} \mathrm{C}$ and a humidity of $40 \%-70 \%$ with free access to food and water. Sterile surgeries were approved by the Animal Care and Use Committee of the First Affiliated Hospital of Guangzhou University of Traditional Chinese Medicine (No. TCMF1-2019004) and accomplished in the SPF laboratory of the First Affiliated Hospital of Guangzhou University of Traditional Chinese Medicine. Humane care was provided to all the rats according to the Guidelines for Ethical Treatment of Experimental Animals published by the Ministry of Science and Technology in 2006. The study was carried out in compliance with the ARRIVE guidelines (http://www.nc3rs.org.uk/page.asp?id=1357).

\section{Model}


On the postnatal day 21 (PND21), the pregnant rats received a 10 min-delayed cesarean section after $12 \mathrm{~h}$ of preoperative fasting. They were weighed first and then placed in a supine position on a surgical board after anesthesia with the abdominal cavity opened along the midline and the uterus exposed. Then, the bilateral uterine aortas were clamped for 10 min before the uterus was cut open and squeezed out in 2 min with their umbilical cords retained; the amniotic fluid in the mouths and noses of the newborn rats was cleaned to promote breathing with dry cotton swabs. Subsequently, the newborn rats were placed on a hot plate covered with moist gauze at $33^{\circ} \mathrm{C}$, and their backs were rubbed repeatedly with a cotton swab moistened with warm physiological saline for rescue. Only the baby rats that could breathe autonomously and were curled up with skin that could turn into pink were selected for our study. Then, the selected baby rats were suckled by the other female rats who delivered naturally. After 21 days of lactation, the male and female offspring were weaned and raised in different cages separately.

\section{Groups}

The neonatal rats underwent a 10 min-delayed cesarean section were assigned to model and acupuncture groups randomly. In the acupuncture group, neonatal rats underwent a 10 min-delayed cesarean section and received an acupuncture treatment $(n=13)$. In the model group, neonatal rats only received a 10 min-delayed cesarean section $(n=13)$. In the normal group, neonatal rats were all delivered naturally $(n=13)$.

\section{Treatments}

Normal group and model group received no treatments but be fixed the same as the other groups. The neonatal rats in acupuncture group received acupuncture treatments. According to Jin's three-needle theory, three acupuncture point groups were selected: the 3-Points for Intelligence (DU24, GB13), the 3Occipital Points (DU17, GB19). The rats were kept awake, and their hair were stroked gently by the intervention staff to make them mentally stable. Beauty acupuncture needles were used $(0.25 \mathrm{~mm} \times 10$ m, Huatuo; Medical Instrument Co., Ltd., Suzhou, China) and acupuncture was applied at the corresponding points with the rat's neck was fixed by index and middle fingers of the left hand. The needles were kept for $10 \mathrm{mins}$ after de qi with the rats placed on the platform $20 \mathrm{~cm}$ away from the ground without environmental interference. The acupuncture treatments were started from PND14 and applied once a day for 2 weeks. What's more, all intervention were accomplished by the same person.

\section{Novel object recognition}

The rats in each group were subjected to a new object recognition experiment after 14 days of acupuncture intervention to observe their ability to recognize old and new objects. An $80 \times 80 \times 40 \mathrm{~cm}$ open box was prepared to have a black bottom and an HD camera $145 \mathrm{~cm}$ directly above it. The bottom of the box was divided into 16 squares of equal size with white lines. The environment was kept dark, and 
the open-field box was clean throughout the experiment. During the adaptation period, the animals were put into an experimental box without any objects for 10 min a day for 3 days continuously. Familiar stage experiments were started on the fourth day, and the rats were adapted to the box for 2 min without any object inside before being taken out. Then, two identical objects (base can be fixed) were put into the box, and the rats were placed into the box again. The exploration times for left objects ( $\mathrm{Tl}$ ) and right objects (Tr) for each rat within 5 min were recorded. After a familiarization period, the rats were put back into the cage, and the test period began 10 min later. During the test period, two different objects were put in the box, one of which was identical to the object from a familiar period and the other was a novel object. Then, the rats were placed in the box for $5 \mathrm{~min}$, and the exploration times for the familiar object (Tf) and novel object $(\mathrm{Tn})$ were recorded. Exploration activities were defined as the rats' noses pointing at objects closely or smelling or licking objects directly. Non-exploratory activity occurred when other parts of the body touched an object without the nose pointing or the rat standing on it.

\section{Nissl staining}

After behavior testing, 3 rats were randomly selected from each group and perfused intracardially with $0.1 \mathrm{~mol} / \mathrm{L}$ PBS and $4 \%$ paraformaldehyde before brains taken out. After fixation in $4 \%$ paraformaldehyde at $4^{\circ} \mathrm{C}$ for $24 \mathrm{~h}$, brains were dehydrated, embedded in paraffin, and then sectioned into a thickness of $4 \mu \mathrm{m}$ for Nissl staining. Morphological observation of brain tissue was performed under high-power light microscopy.

\section{RNA isolation, library preparation, and sequencing}

Total RNA was extracted from brain samples (prefrontal lobe) with Trizol reagent (Invitrogen) according to the manufacturer's instructions. RNA degradation and contamination were monitored on $1 \%$ agarose gels. The RNA purity, concentrations and integrity were assessed by NanoPhotometer ${ }^{\circledR}$ spectrophotometer (IMPLEN, CA, USA), Qubit ${ }^{\circledR}$ RNA Assay Kit in Qubit ${ }^{\circledR} 2.0$ Fluorometer (Life Technologies, CA, USA) and RNA Nano 6000 Assay Kit for a Bioanalyzer 2100 system (Agilent Technologies, CA, USA).

A total of $3 \mu \mathrm{g}$ of RNA per sample was used as input material for the RNA sample preparations. Under the manufacturer's recommendations, NEBNext ${ }^{\circledR}$ UltraTM RNA Library Prep Kit for Illumina ${ }^{\circledR}$ (NEB, USA) was used to generate the sequencing libraries with index codes added to each sample. Briefly, mRNA was purified from the total RNA using poly-T oligo-attached magnetic beads. The RNA fragmentation, first and second strand cDNA synthesis, end repair, adaptor connection and PCR amplification were conducted with the manufacturer's protocol. After the library was constructed, Qubit 2.0 and Agilent 2100 were first used for validation of RNA integrity and quantity. Subsequently, qRT-PCR was performed to accurately quantify the effective concentration of the library (the effective concentration of the library was higher than $3 \mathrm{~nm}$ ) to ensure the quality of the library. 
According to the manufacturer's protocol, TruSeq PE Cluster Kit v3-cBot-HS (Illumina) was used to cluster of the index-coded samples on the cBot Cluster Generation System. the library preparations were sequenced on an Illumina Hiseq platform after cluster generation, and $125 \mathrm{bp} / 150 \mathrm{bp}$ paired-end reads were generated.

\section{Quality control, mapping, and quantification}

Raw data were first processed through in-house perl scripts. Reads containing adapter, ploy-N, and lowquality reads were removed from raw data to obtain clean reads. At the same time, Q20, Q30, and the GC content of the clean data were calculated (Table S1). All the succeeding analyses were based on the clean data with high quality. STAR (v2.5.1b) was used to build the index of the reference genome downloaded from the genome website directly and to connect paired-end clean reads to the reference genome (Table S2). HTSeq v0.6.0 was used to count the reads mapped to each gene. Then, the FPKM of each gene was calculated to estimate gene expression levels (Table S3).

\section{LncRNA functional prediction and expression analysis}

Cis and trans genes of IncRNA were detected for further functional analysis. The cis gene is IncRNA that acts on adjacent target genes. The coding genes 10k/100k upstream and downstream of IncRNA were searched and analyzed for their function. The trans gene is IncRNA that can be used to identify each other by the expression level. The expressed correlation between coding genes and IncRNAs were calculated with custom scripts. Cuffdiff (v2.1.1) was used to calculate FPKMs of both IncRNAs and coding genes in each sample.

\section{GO and KEGG enrichment analysis}

Gene Ontology (GO) enrichment analysis of differentially expressed genes was carried out by the cluster Profiler R package. The cluster Profiler R package was applied to test the statistical enrichment of differentially expressed genes in Kyoto Encyclopedia of Genes and Genomes (KEGG) pathways for further understanding of high-level functions and utilities of biological systems (http://www.genome.jp/kegg/).

\section{Statistical analysis}

No statistical methods were used to determine the experimental sample size. The sample size (Biologically independent rats, n), $p$-values and types of statistical tests are marked on diagrams or legends. SPSS 23.0 was used for statistical analysis. Continuous data are expressed as mean \pm standard deviation ( $\mathrm{x} \pm \mathrm{s}$ ). Paired t test was used to compare TL and TR or TF and TN within groups. Relative Discrimination Index (DI) was calculated according to the formula $\mathrm{Di}=(\mathrm{TN}-\mathrm{TF}) /(\mathrm{TN}+\mathrm{TF})$, that is, the 
proportion of the exploration time of new object more than familiar object in the total exploration time in the test period. One-way ANOVA was used to compare the mean values of multiple groups. KolmogorovSmirnov $(\mathrm{n}<50)$ was used to test for normality. Data subject to normal distribution was analyzed by Levene Statistic. If the variances were uniform, the independent sample t-test or LSD test was used, and the difference was statistically significant according to the level of $a=0.05, P<0.05$. Statistical graphs were processed using GraphPad Prism 8. Statistical analysis was completed by the researcher who was not involved in the experiments.

Cuffdiff (v2.1.1) was used to evaluate the expression levels of IncRNAs in the transcripts of each sample, whereas DEGseq was applied to assess the expression levels of circRNAs of each sample. The results with a corrected $P<0.05$ were considered to be statistically significant. Pearson's correlation coefficient was applied for the correlation analysis between IncRNAs and target mRNAs. If the absolute value of Pearson's coefficient was $>0.95$, the targeted relations were assigned to be statistically significant. The GO terms enriched by differentially expressed genes were statistically significant with corrected $P<0.05$. The results of the enrichment of KEGG pathways were evaluated by KOBAS and were considered to be statistically significant with $P<0.05$.

\section{Declarations}

\section{Acknowledgements}

This research was funded by the National Natural Science Foundation of China (NO. 81973950).

\section{Author Contributions}

W.Q. conceived and designed the experiments. W.Q., J.W., J.X., Y.Z. and C.Y. performed the experiments. X.J. analyzed the results. X.J. and W.Q drafted the manuscript. Q.Y. revised the manuscript. All authors read and approved the final manuscript.

\section{Competing financial interests:}

The authors declare no competing financial interests.

\section{References}

1. Yang, L., Zhao, H. \& Cui, H. Treatment and new progress of neonatal hypoxic-ischemic brain damage. Histology and histopathology, 18214, doi:10.14670/hh-18-214 (2020).

2. Vannucci, S. J. \& Hagberg, H. Hypoxia-ischemia in the immature brain. The Journal of experimental biology 207, 3149-3154, doi:10.1242/jeb.01064 (2004). 
3. du Plessis, A. J. \& Volpe, J. J. Perinatal brain injury in the preterm and term newborn. Current opinion in neurology 15, 151-157, doi:10.1097/00019052-200204000-00005 (2002).

4. Celik, Y. et al. Comparison of selective head cooling versus whole-body cooling. Pediatrics international : official journal of the Japan Pediatric Society 58, 27-33, doi:10.1111/ped.12747 (2016).

5. Aly, H. et al. Melatonin use for neuroprotection in perinatal asphyxia: a randomized controlled pilot study. Journal of perinatology : official journal of the California Perinatal Association 35, 186-191, doi:10.1038/jp.2014.186 (2015).

6. Huang, X. et al. [Clinical efficacy on mental retardation in the children treated with JIN's three scalp needling therapy and the training for cognitive and perceptual disturbance]. Zhongguo zhen jiu = Chinese acupuncture \& moxibustion 35, 651-656 (2015).

7. Yuan, Q, et al., Effect of synchronous training with needle retention on cognitive ability of children with mental retardation. J Guangzhou University of Chinese Medicine, 2013. 30(05): p. 672-676..

8. Yuan, Q. et al. [Treatment of autism children: observation on efficacy of behavior training with retention of needles on head]. Zhongguo zhen jiu = Chinese acupuncture \& moxibustion 33, 609-613 (2013).

9. Luo, Q \& Luo, Q. Observation on the clinical effect of Jin's 3 needle on attention deficit hyperactivity disorder. World Chin Med 6, 230-232 (2011).

10. Li, $\mathrm{S}$ et al., Study on the mechanism of acupuncture on hypoxic-ischemic brain injury in rats with intrauterine distress. Liaoning J Trad Chin Med, 45, 842-845 (2018).

11. Yuan, $Q$ et al., The effect on the expression of Cyt-C and Caspase-3 in cerebral cortex of intrauterine distress in HIBD rats treated by Jin's three needles. Shanghai Journal of Acupuncture ,34, 794-797 (2015).

12. Yuan, $Q$, et al., the effects of apoptosis on the ratio of Bcl-2 and Bax in HIBD rats treated by Jin's three needles. Liaoning J Trad Chin Med, 42, 202-204 (2015).

13. Zhang, F., Zhang, L. \& Zhang, C. Long noncoding RNAs and tumorigenesis: genetic associations, molecular mechanisms, and therapeutic strategies. Tumour biology : the journal of the International Society for Oncodevelopmental Biology and Medicine 37, 163-175, doi:10.1007/s13277-015-4445-4 (2016).

14. Li, T., Mo, X., Fu, L., Xiao, B. \& Guo, J. Molecular mechanisms of long noncoding RNAs on gastric cancer. Oncotarget 7, 8601-8612, doi:10.18632/oncotarget.6926 (2016).

15. Fu, M. et al. Long noncoding RNAs in digestive system cancers: Functional roles, molecular mechanisms, and clinical implications (Review). Oncology reports 36, 1207-1218, doi:10.3892/or.2016.4929 (2016).

16. Clark, B. S. \& Blackshaw, S. Understanding the Role of IncRNAs in Nervous System Development. Advances in experimental medicine and biology 1008, 253-282, doi:10.1007/978-981-10-5203-3_9 (2017). 
17. Zhao, R. B., Zhu, L. H., Li, H. J., Fan, Z. M. \& Xia, Z. K. High-throughput sequencing analysis of IncRNAs in hippocampus tissues with hypoxic-ischemic brain damage. International journal of clinical and experimental pathology 11, 5265-5277 (2018).

18. Li, H. et al. The role of a IncRNA (TCONS_00044595) in regulating pineal CLOCK expression after neonatal hypoxia-ischemia brain injury. Biochemical and biophysical research communications 528 , 1-6, doi:10.1016/j.bbrc.2020.05.047 (2020).

19. Zhao, F. et al. Microarray Profiling and Co-Expression Network Analysis of LncRNAs and mRNAs in Neonatal Rats Following Hypoxic-ischemic Brain Damage. Scientific reports 5, 13850, doi:10.1038/srep13850 (2015).

20. Raith, W., Urlesberger, B. \& Schmölzer, G. M. Efficacy and safety of acupuncture in preterm and term infants. Evidence-based complementary and alternative medicine : eCAM 2013, 739414, doi:10.1155/2013/739414 (2013).

21. Yang, C., Hao, Z., Zhang, L. L. \& Guo, Q. Efficacy and safety of acupuncture in children: an overview of systematic reviews. Pediatric research 78, 112-119, doi:10.1038/pr.2015.91 (2015).

22. Yi, W., Xu, N. G. \& Wang, G. B. [Experimental study on effects of electro-acupuncture in improving synaptic plasticity in focal cerebral ischemia rats]. Zhongguo Zhong xi yi jie he za zhi Zhongguo Zhongxiyi jiehe zazhi $=$ Chinese journal of integrated traditional and Western medicine 26, 710-714 (2006).

23. Gao, J., Wang, S., Wang, X. \& Zhu, C. Electroacupuncture enhances cell proliferation and neuronal differentiation in young rat brains. Neurological sciences : official journal of the Italian Neurological Society and of the Italian Society of Clinical Neurophysiology 32, 369-374, doi:10.1007/s10072-0100402-6 (2011).

24. Li, M. et al. Electro-acupuncture combined with transcranial magnetic stimulation improves learning and memory function of rats with cerebral infarction by inhibiting neuron cell apoptosis. Journal of Huazhong University of Science and Technology. Medical sciences = Hua zhong ke ji da xue xue bao. Yi xue Ying De wen ban = Huazhong keji daxue xuebao. Yixue Yingdewen ban 32, 746-749, doi:10.1007/s11596-012-1028-0 (2012).

25. Zhou, H. et al. Analysis of long non-coding RNA expression profiles in neonatal rats with hypoxicischemic brain damage. Journal of neurochemistry 149, 346-361, doi:10.1111/jnc.14689 (2019).

26. Jiang, L., Li, H., Fan, Z., Zhao, R. \& Xia, Z. Circular RNA expression profiles in neonatal rats following hypoxic-ischemic brain damage. International journal of molecular medicine 43, 1699-1708, doi:10.3892/ijmm.2019.4111 (2019).

27. Jiang, W. et al. Vitamin A bio-modulates apoptosis via the mitochondrial pathway after hypoxicischemic brain damage. Mol Brain 11, 14, doi:10.1186/s13041-018-0360-0

28. Wei, L., Ren, Q., Zhang, Y. \& Wang, J. Effects of hyperbaric oxygen and nerve growth factor on the long-term neural behavior of neonatal rats with hypoxic ischemic brain damage. Acta Cir Bras 32, 270-279, doi:S0102-86502017000400270 [pii]10.1590/s0102-865020170040000002 [doi] (2017). 
29. Wang, X. L. et al. [Effect of hyperbaric oxygen therapy administered at different time on white matter damage following hypoxic-ischemic brain damage in neonatal rats]. Zhongguo Dang Dai Er Ke Za Zhi 9, 308-312, doi:1008-8830(2007)04-0308-05 [pii] (2007).

30. Li, Z. H. \& Chen, C. [Analysis of 58 neonatal cases with cerebral infarction]. Zhonghua Er Ke Za Zhi $51,16-20$ (2013).

31. Spasojevic, S. D. et al. Neuroprotective effects of hypothermia and erythropoietin after perinatal asphyxia in newborn rats. J Matern Fetal Neonatal Med 26, 1506-1509, doi:10.3109/14767058.2013.789846 [doi] (2013).

32. Xin, T., Li, Z. \& Zhang, X. Use of MRI and CT image indexing to assess cerebral injuries in neonates with hypoxic-ischemic encephalopathy. Minerva Pediatr 71, 438-442, doi:R15Y9999N00A150042 [pii]10.23736/S0026-946.16.04349-8 [doi] (2019).

33. Gao J, et al., Acupuncture improves locomotor activity and learning-memory ability by improving hippocampal cellular autophagy in rats with fetal intrauterine distress. Zhen Ci Yan Jiu, 45, 275$280+324(2020)$.

34. You, Y. H., Qin, Z. Q., Zhang, H. L., Yuan, Z. H. \& Yu, X. MicroRNA-153 promotes brain-derived neurotrophic factor and hippocampal neuron proliferation to alleviate autism symptoms through inhibition of JAK-STAT pathway by LEPR. Bioscience reports 39, doi:10.1042/bsr20181904 (2019).

35. Qin, H. et al. Inhibition of the JAK/STAT Pathway Protects Against a-Synuclein-Induced Neuroinflammation and Dopaminergic Neurodegeneration. The Journal of neuroscience : the official journal of the Society for Neuroscience 36, 5144-5159, doi:10.1523/jneurosci.4658-15.2016 (2016).

36. Tao, W., Wen, F., Zhang, H. \& Liu, G. The signal transduction mediated by erythropoietin and proinflammatory cytokines in the JAK/STAT pathway in the children with cerebral palsy. Brain \& development 31, 200-207, doi:10.1016/j.braindev.2008.06.011 (2009).

37. Zhang, Z., Yan, J., Taheri, S., Liu, K. J. \& Shi, H. Hypoxia-inducible factor 1 contributes to Nacetylcysteine's protection in stroke. Free radical biology \& medicine 68, 8-21, doi:10.1016/j.freeradbiomed.2013.11.007 (2014).

38. Zhang, B., Wu, H. Q., Zhang, H. X., Zhang, G. L. \& Zhan, S. Q. [A study on expression of HIF-1alpha and EPO in the hippocampus of rats with vascular dementia]. Sichuan da xue xue bao. Yi xue ban = Journal of Sichuan University. Medical science edition 37, 730-733 (2006).

39. Guo, M. et al. In chronic hypoxia, glucose availability and hypoxic severity dictate the balance between HIF-1 and HIF-2 in astrocytes. FASEB journal : official publication of the Federation of American Societies for Experimental Biology 33, 11123-11136, doi:10.1096/fj.201900402RR (2019).

40. Cheng, C. Y. et al. Angelica sinensis Exerts Angiogenic and Anti-apoptotic Effects Against Cerebral Ischemia-Reperfusion Injury by Activating p38MAPK/HIF-1[Formula: see text]/VEGF-A Signaling in Rats. The American journal of Chinese medicine 45, 1683-1708, doi:10.1142/s0192415x17500914 (2017).

41. Wu, Q. \& Yi, X. Down-regulation of Long Noncoding RNA MALAT1 Protects Hippocampal Neurons Against Excessive Autophagy and Apoptosis via the PI3K/Akt Signaling Pathway in Rats with 
Epilepsy. Journal of molecular neuroscience : MN 65, 234-245, doi:10.1007/s12031-018-1093-3 (2018).

42. Yu, D. F., Zhu, L. H. \& Jiang, L. Recombinant Human Erythropoietin Augments Neovascularization Responses in a Neonatal Rat Model of Premature Brain Damage by Phosphatidylinositol 3 Kinase/Akt Pathway. Chinese medical journal 130, 854-858, doi:10.4103/0366-6999.202744 (2017).

43. Perluigi, M., Barone, E., Di Domenico, F. \& Butterfield, D. A. Aberrant protein phosphorylation in Alzheimer disease brain disturbs pro-survival and cell death pathways. Biochimica et biophysica acta 1862, 1871-1882, doi:10.1016/j.bbadis.2016.07.005 (2016).

44. England, B., Huang, T. \& Karsy, M. Current understanding of the role and targeting of tumor suppressor p53 in glioblastoma multiforme. Tumour biology : the journal of the International Society for Oncodevelopmental Biology and Medicine 34, 2063-2074, doi:10.1007/s13277-013-0871-3 (2013).

45. Uzdensky, A. B. Apoptosis regulation in the penumbra after ischemic stroke: expression of pro- and antiapoptotic proteins. Apoptosis : an international journal on programmed cell death $24,687-702$, doi:10.1007/s10495-019-01556-6 (2019).

46. Bhattacharyya, S. et al. CDKN2A-p53 mediated antitumor effect of Lupeol in head and neck cancer. Cellular oncology (Dordrecht) 40, 145-155, doi:10.1007/s13402-016-0311-7 (2017).

47. Neurath, M. F. \& Finotto, S. IL-9 signaling as key driver of chronic inflammation in mucosal immunity. Cytokine \& growth factor reviews 29, 93-99, doi:10.1016/j.cytogfr.2016.02.002 (2016).

48. Donninelli, G. et al. Interleukin-9 regulates macrophage activation in the progressive multiple sclerosis brain. Journal of neuroinflammation 17, 149, doi:10.1186/s12974-020-01770-z (2020).

49. England, T. J., Gibson, C. L. \& Bath, P. M. Granulocyte-colony stimulating factor in experimental stroke and its effects on infarct size and functional outcome: A systematic review. Brain research reviews 62, 71-82, doi:10.1016/j.brainresrev.2009.09.002 (2009).

50. Casarini, L. \& Crépieux, P. Molecular Mechanisms of Action of FSH. Frontiers in endocrinology 10 , 305, doi:10.3389/fendo.2019.00305 (2019).

51. Weinstein, J. J. et al. Pathway-Specific Dopamine Abnormalities in Schizophrenia. Biological psychiatry 81, 31-42, doi:10.1016/j.biopsych.2016.03.2104 (2017).

52. Masato, A., Plotegher, N., Boassa, D. \& Bubacco, L. Impaired dopamine metabolism in Parkinson's disease pathogenesis. Molecular neurodegeneration 14, 35, doi:10.1186/s13024-019-0332-6 (2019).

53. Nakashima, A. et al. Identification by nano-LC-MS/MS of NT5DC2 as a protein binding to tyrosine hydroxylase: Down-regulation of NT5DC2 by siRNA increases catecholamine synthesis in PC12D cells. Biochemical and biophysical research communications 516, 1060-1065, doi:10.1016/j.bbrc.2019.06.156 (2019).

54. Salvatore, M. F., Mclnnis, T. R., Cantu, M. A., Apple, D. M. \& Pruett, B. S. Tyrosine Hydroxylase Inhibition in Substantia Nigra Decreases Movement Frequency. Molecular neurobiology 56, 27282740, doi:10.1007/s12035-018-1256-9 (2019). 
55. Vengeliene, V. et al. Towards trans-diagnostic mechanisms in psychiatry: neurobehavioral profile of rats with a loss-of-function point mutation in the dopamine transporter gene. Disease models \& mechanisms 10, 451-461, doi:10.1242/dmm.027623 (2017).

56. McCoy, M. K. \& Tansey, M. G. TNF signaling inhibition in the CNS: implications for normal brain function and neurodegenerative disease. Journal of neuroinflammation 5,45 , doi:10.1186/17422094-5-45 (2008).

57. Kim, Y. K., Shin, J. S. \& Nahm, M. H. NOD-Like Receptors in Infection, Immunity, and Diseases. Yonsei medical journal 57, 5-14, doi:10.3349/ymj.2016.57.1.5 (2016).

58. Liu, F., Li, Z., He, X., Yu, H. \& Feng, J. Ghrelin Attenuates Neuroinflammation and Demyelination in Experimental Autoimmune Encephalomyelitis Involving NLRP3 Inflammasome Signaling Pathway and Pyroptosis. Frontiers in pharmacology 10, 1320, doi:10.3389/fphar.2019.01320 (2019).

59. Gamdzyk, M. et al. Role of PPAR- $\beta / \delta / m i R-17 / T X N I P$ pathway in neuronal apoptosis after neonatal hypoxic-ischemic injury in rats. Neuropharmacology 140, 150-161, doi:10.1016/j.neuropharm.2018.08.003 (2018).

60. Sahoo, A. et al. A novel splicing variant of mouse interleukin (IL)-24 antagonizes IL-24-induced apoptosis. The Journal of biological chemistry 283, 28860-28872, doi:10.1074/jbc.M802510200 (2008).

61. Ampuero, E., Jury, N., Härtel, S., Marzolo, M. P. \& van Zundert, B. Interfering of the Reelin/ApoER2/PSD95 Signaling Axis Reactivates Dendritogenesis of Mature Hippocampal Neurons. Journal of cellular physiology 232, 1187-1199, doi:10.1002/jcp.25605 (2017).

62. Bender, R. A. et al. Roles of $17 ß$-estradiol involve regulation of reelin expression and synaptogenesis in the dentate gyrus. Cerebral cortex (New York, N.Y. : 1991) 20, 2985-2995, doi:10.1093/cercor/bhq047 (2010).

63. Rideau Batista Novais, A. et al. N-acetyl-cysteine prevents pyramidal cell disarray and reelinimmunoreactive neuron deficiency in CA3 after prenatal immune challenge in rats. Pediatric research 73, 750-755, doi:10.1038/pr.2013.40 (2013).

64. Sui, L., Wang, Y., Ju, L. H. \& Chen, M. Epigenetic regulation of reelin and brain-derived neurotrophic factor genes in long-term potentiation in rat medial prefrontal cortex. Neurobiology of learning and memory 97, 425-440, doi:10.1016/j.nlm.2012.03.007 (2012).

65 . Beffert, $U$. et al. Reelin-mediated signaling locally regulates protein kinase B/Akt and glycogen synthase kinase 3beta. The Journal of biological chemistry 277, 49958-49964, doi:10.1074/jbc.M209205200 (2002).

\section{Figures}


A

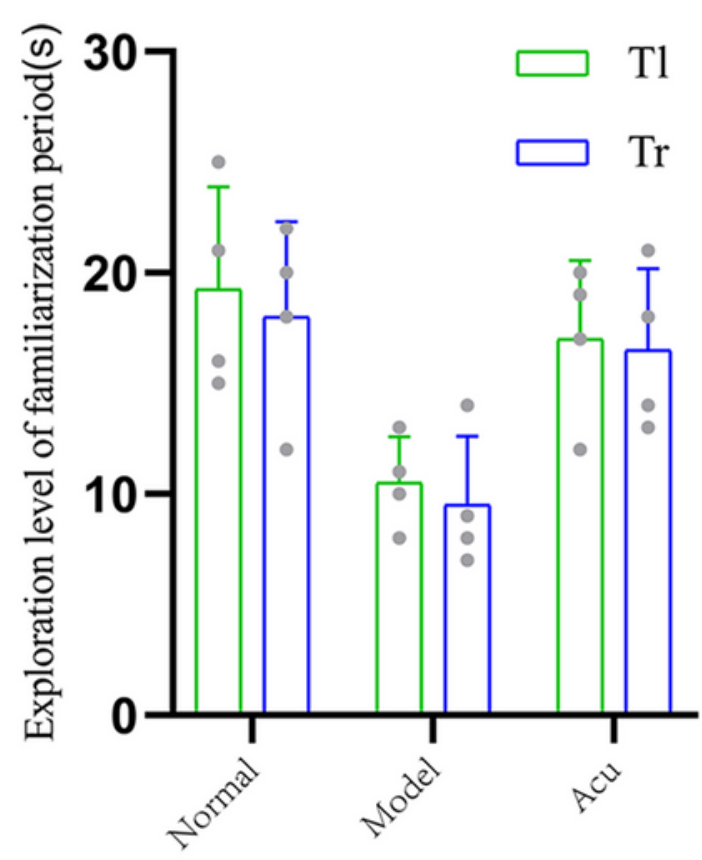

B

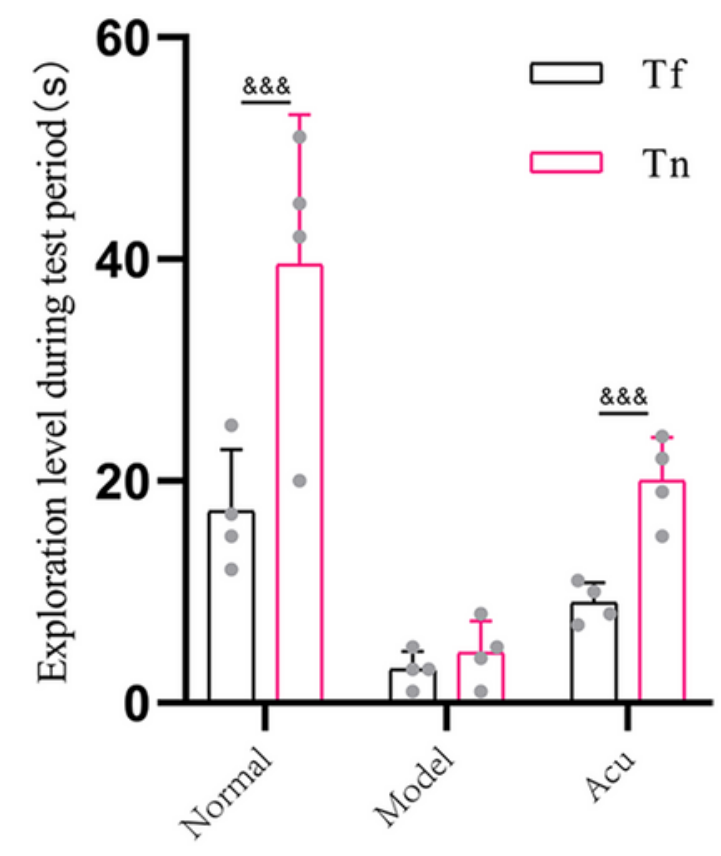

C

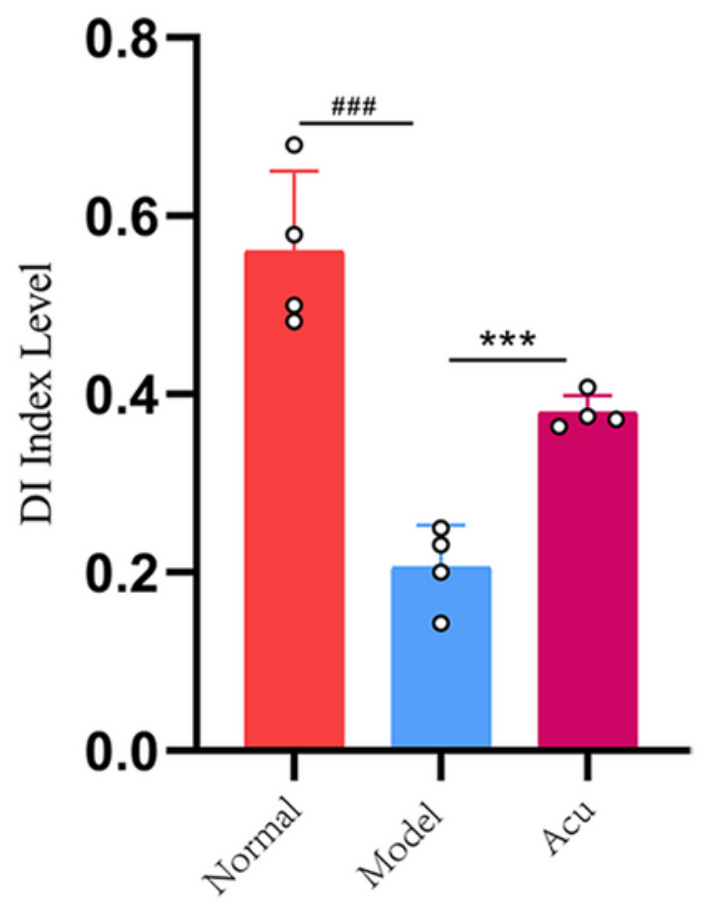

Figure 1

Effect of acupuncture on new object recognition in HIBD rats. (A) Exploration level of the familiarity period in a new object recognition experiment in each group of rats $(n=13)$; (B) exploration levels in the test period for new object recognition in each group of rats(n=13); (C) DI level of new object recognition experiment in the rats of each group $(n=13)$; compared with the normal group, \#\#\#P<0.001, \#\#P<0.01. Compared with the model group, ${ }^{\star \star *} \mathrm{P}<0.001$. Compared with sham acupuncture group, \&\&\&P<0.001. 


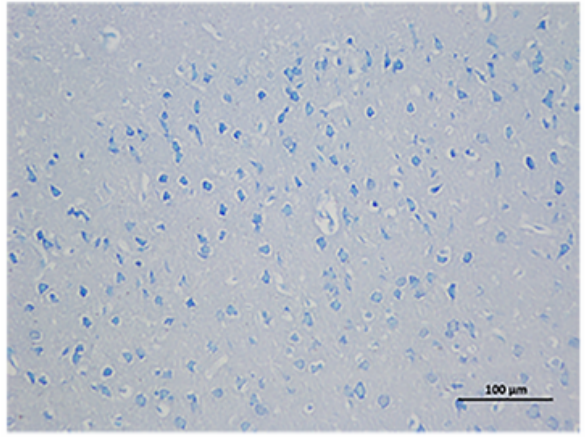

Normal

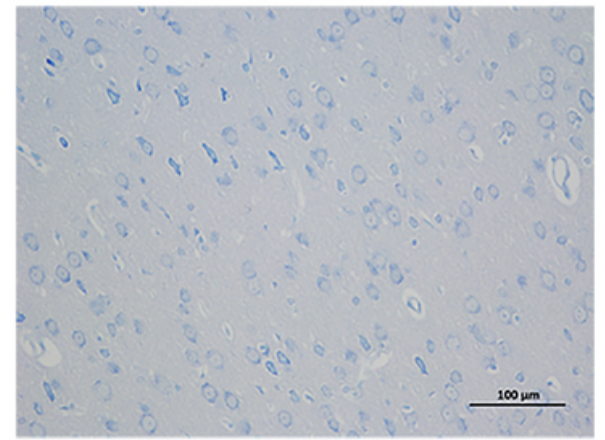

Model

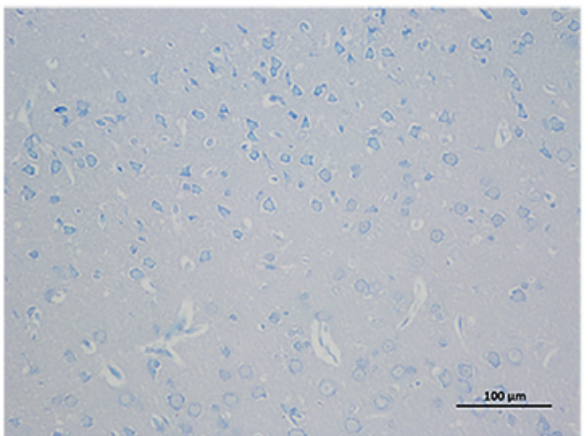

Acupuncture

Figure 2

Pathological changes of prefrontal lobe in each group of rats with Nissl staining. Representative prefrontal sections with Nissl staining of rats in normal group (left, $n=3$ ), model group (middle, $n=3$ ) and acupuncture group (right, $n=3$ ). Scale bar $=100 \mu \mathrm{m}$.

A

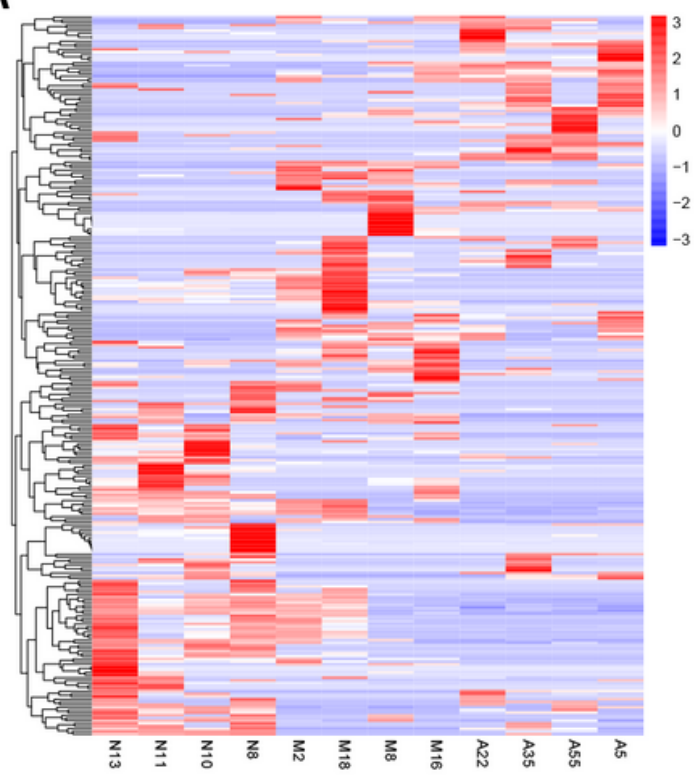

B

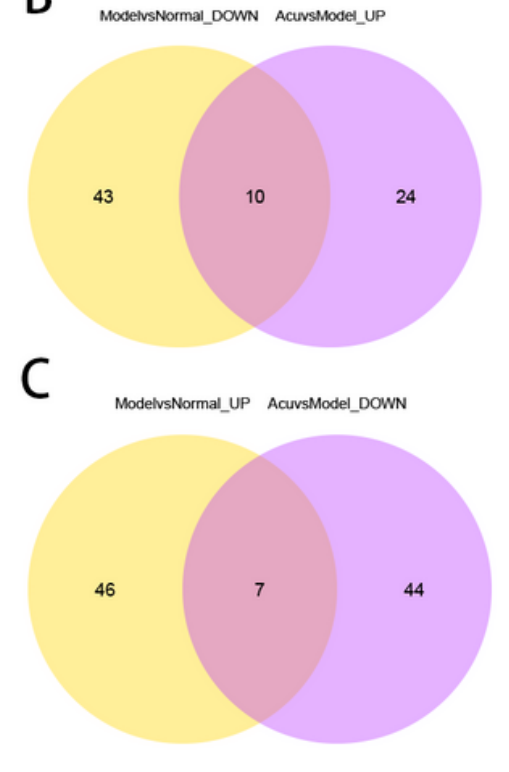

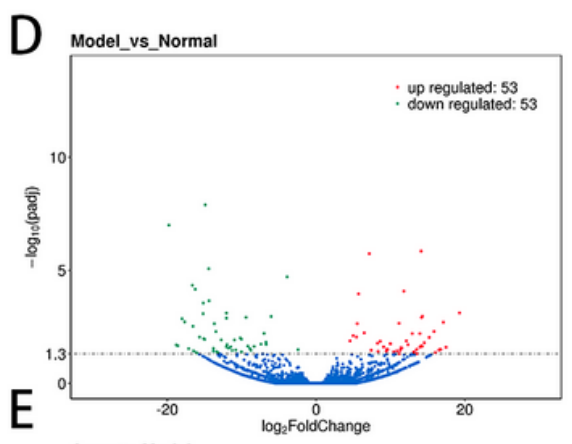

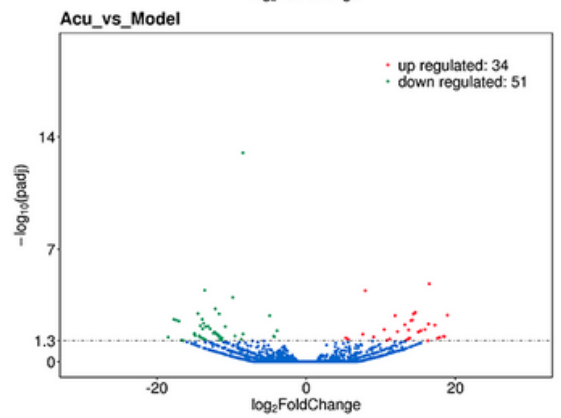

\section{Figure 3}

Identification and characteristic comparisons of mRNAs. (A) Heat map of differentially expressed mRNAs between the samples of the acupuncture, model, and normal groups $(n=4, P<0.05)$. The spectrum color of blue represents down-regulated, whereas red represents up-regulated. N1-4 represents four samples of the normal group. M1-4 represents four samples of the model group. A1-4 represents the four samples of the acupuncture group. $(B, C)$ Volcano plots demonstrate that 133 mRNAs were significantly and differentially expressed in the model rats relative to the normal rats with 97 up-regulated and 36 down- 
regulated; 48 mRNAs were significantly and differentially expressed in the rats that received acupuncture relative to the model rats with 7 up-regulated and 41 down-regulated. (D, E) The Venn diagrams show the overlap of differentially expressed mRNAs between the experimental groups.

A

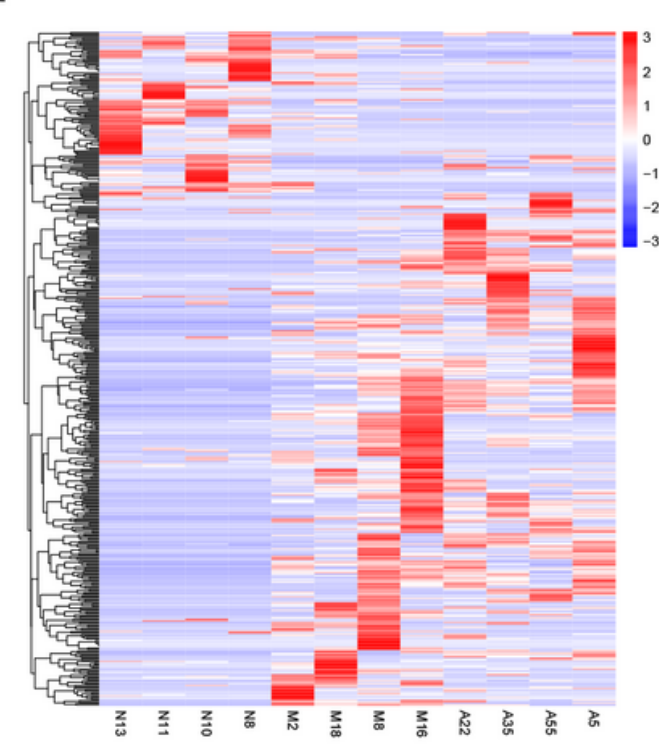

B

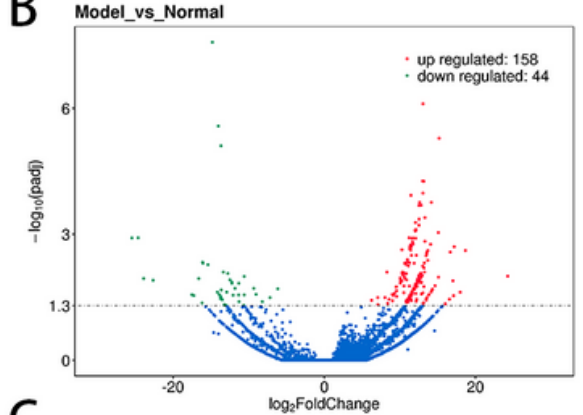

C

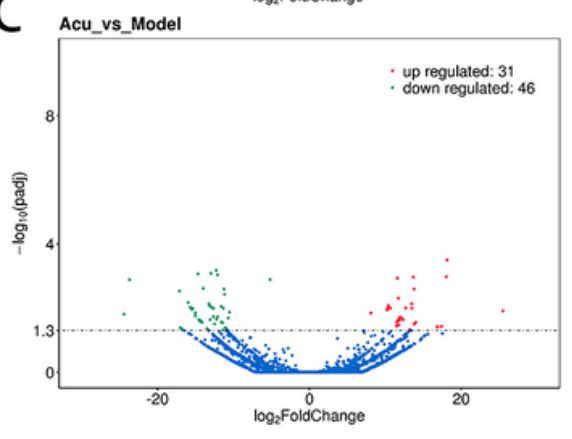

D

ModelvsNormal_DOWN AcuvsModel_UP

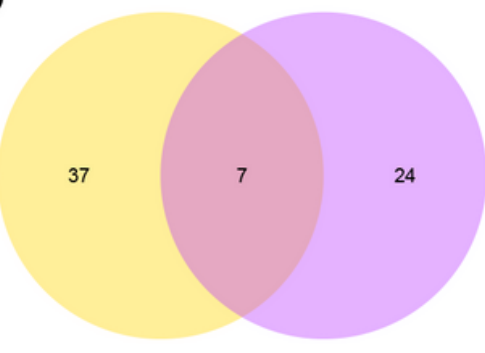

$\mathrm{E}$

ModelvsNormal_UP AcursModel_DOWN

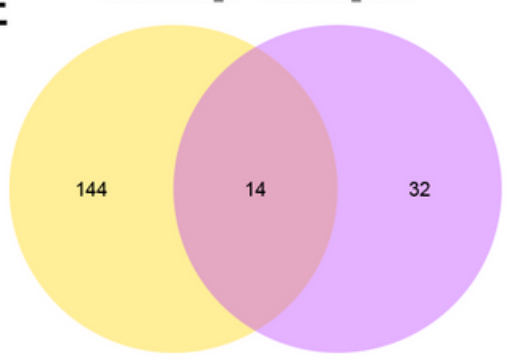

\section{Figure 4}

Identification and characteristic comparison of IncRNAs. (A) Heat map of differentially expressed IncRNAs between the experimental groups $(n=4, P<0.05)$. The spectrum color of blue represents the down-regulated, whereas red represents the up-regulated. N1-4 represents the four samples of the normal group. M1-4 represents four samples of the model group. A1-A4 represents four samples of the acupuncture group. (B, C) Volcano plots demonstrated that 410 IncRNAs were significantly and differentially expressed in the model rats relative to the normal rats with 372 up-regulated and 38 downregulated; 65 IncRNAs were significantly and differentially expressed in the rats that received acupuncture relative to the model rats with 17 up-regulated and 48 down-regulated. (D, E) The Venn diagrams show the overlap of differentially expressed IncRNAs between the experimental groups.
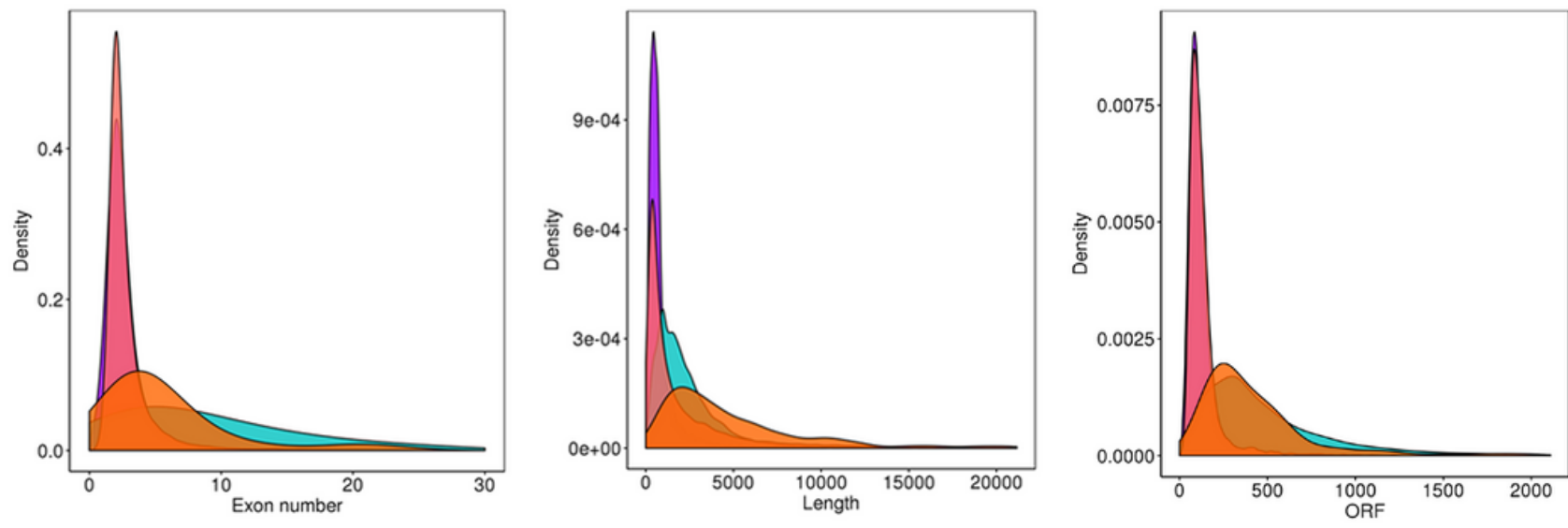


\section{Figure 5}

The comparison of characteristics between mRNAs and IncRNAs demonstrated that IncRNAs had fewer exons, shorter lengths, and shorter lengths of the open reading frame (ORF) than mRNAs.

A

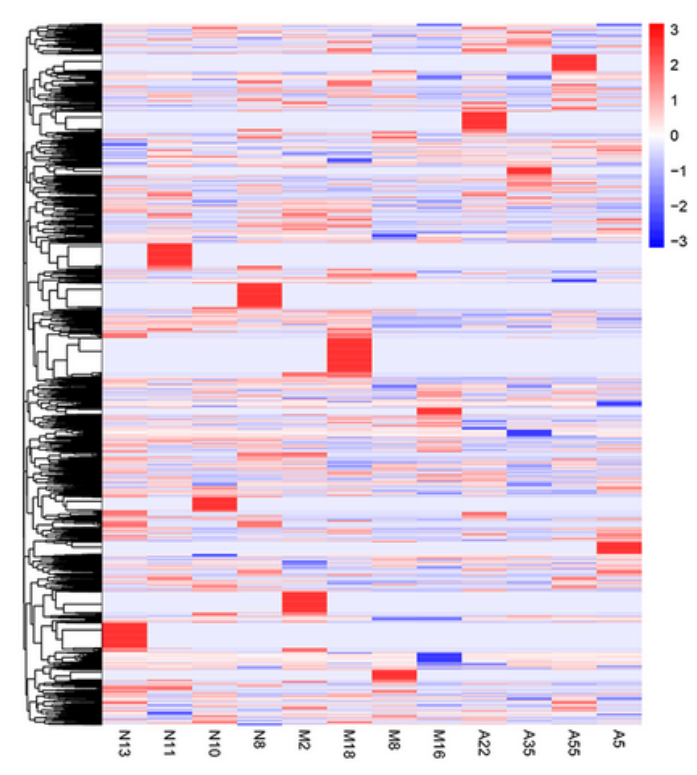

B

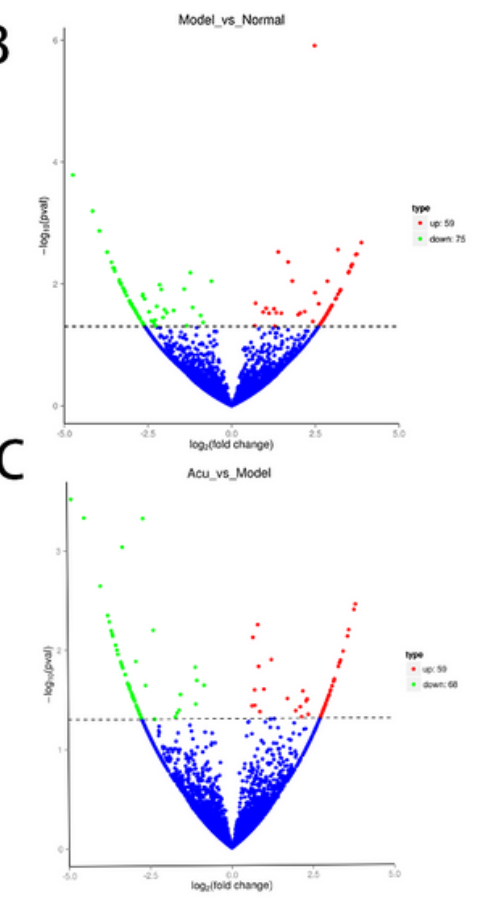

D

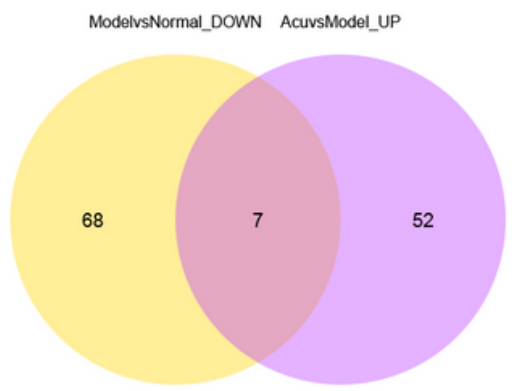

$\mathrm{E}$

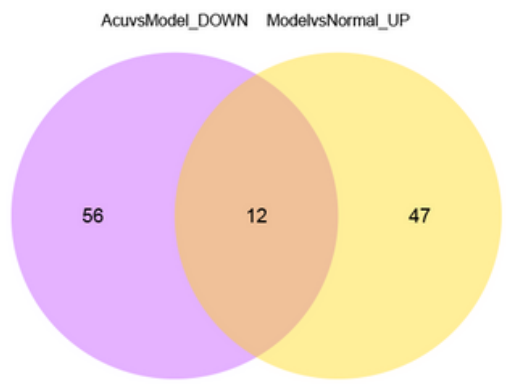

Figure 6

Identification and characteristic comparison of circRNAs. (A) Heat map of differentially expressed circRNAs between the experimental groups $(n=4, P<0.05)$. The spectrum color of blue represents downregulated, whereas red represents up-regulated. N1-4 represents four samples of the normal group. M1-4 represented four samples of the model group. A1-A4 represents four samples of the acupuncture group. (B, C) Volcano plots demonstrated that 134 circRNAs were significantly and differentially expressed in the model rats relative to the normal rats with 59 up-regulated and 75 down-regulated; 127 circRNAs were significantly and differentially expressed in the rats that received acupuncture relative to the model rats with 59 up-regulated and 68 down-regulated. (D, E) The Venn diagrams show the overlapping differentially expressed circRNAs between the experimental groups. 
A

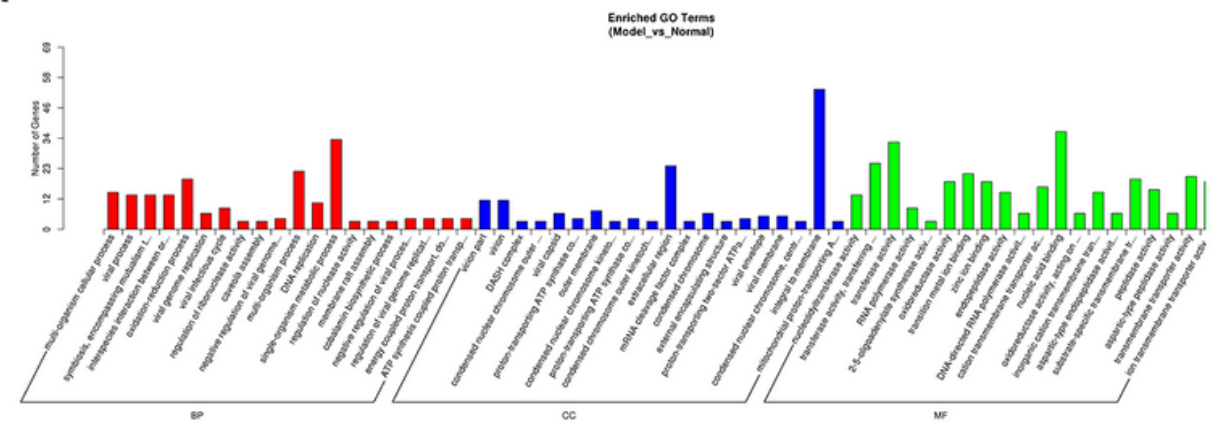

B

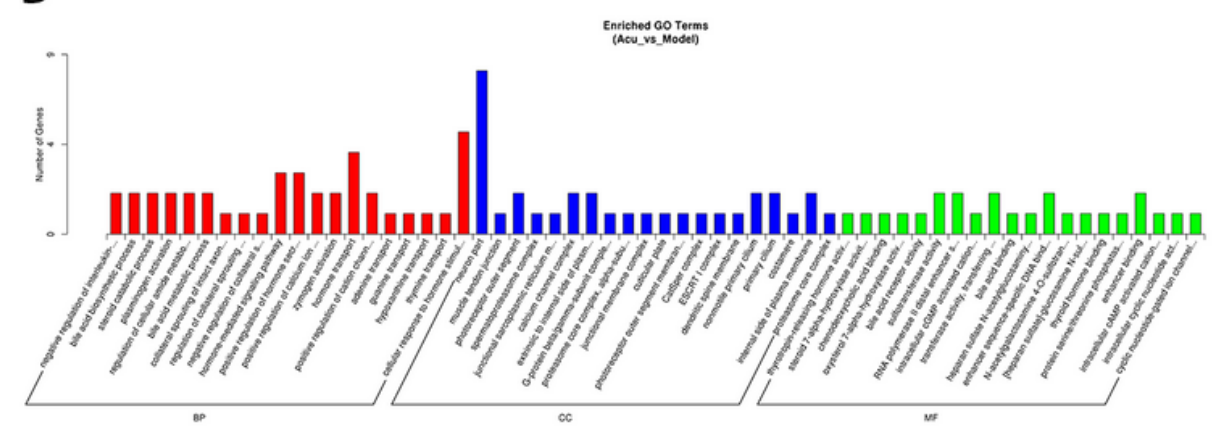

C
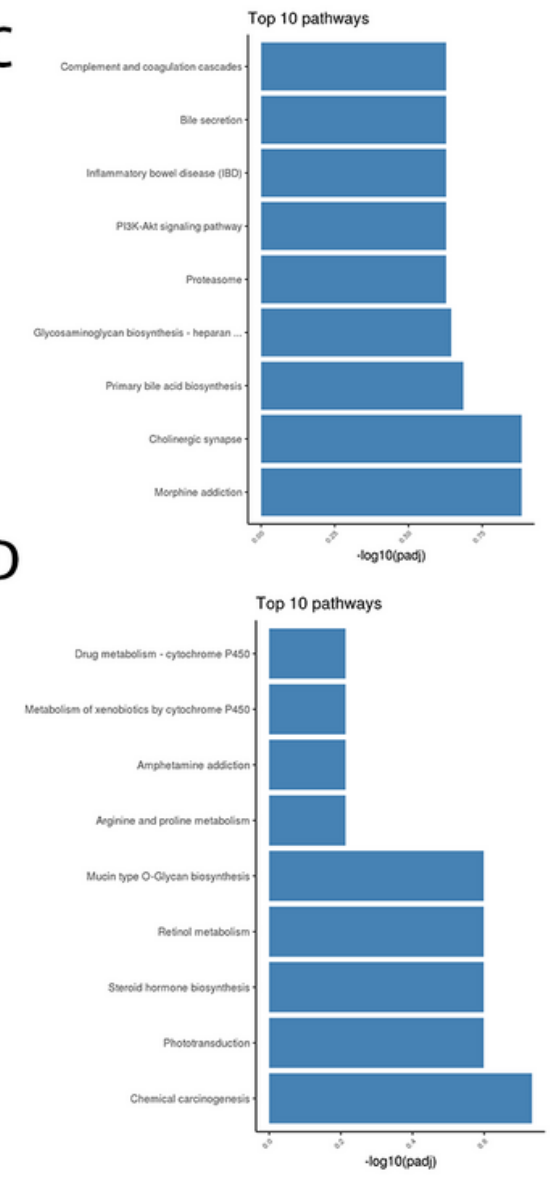

Figure 7

Bioinformatics analysis of the mRNAs. The 60 most significant $\mathrm{GO}$ terms of mRNAs that were differentially expressed across the domains of biological processes (BP), cellular components (CC), and molecular functions (MF). GO, Gene Ontology; Acu, acupuncture group; Model, model group; Normal, Normal group. The vertical coordinates on the left represent the number of mRNAs. (A) The significant GO terms of mRNAs that were differentially expressed between the model and normal groups. (B) The significant GO terms of mRNAs that were differentially expressed between the acupuncture group and model group. (C) The 10 most prominent KEGG pathways of the comparison between acupuncture and model groups. (D) The 10 most prominent KEGG pathways in the comparison between model and acupuncture groups. 
A

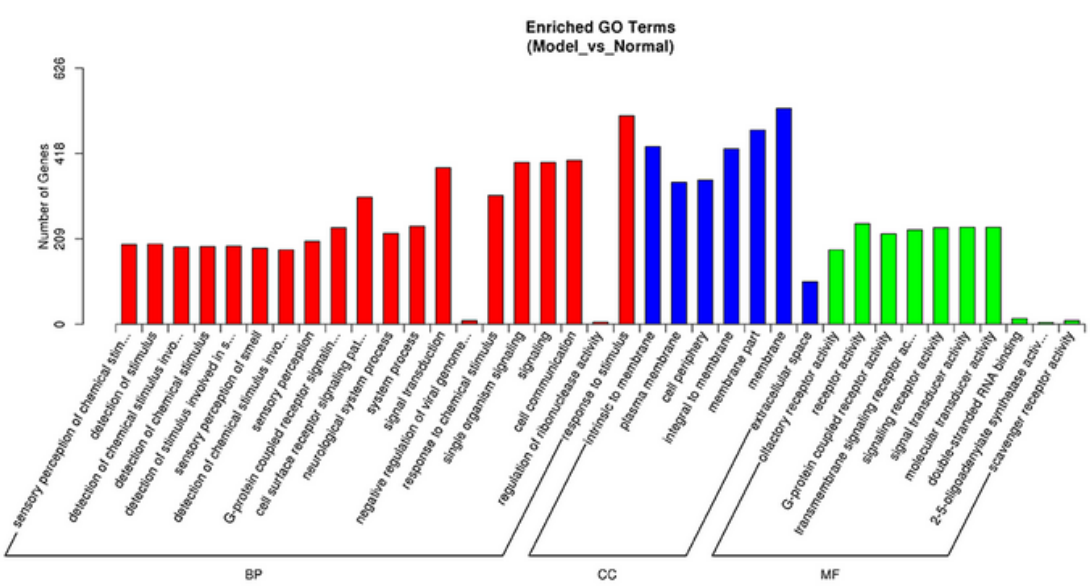

B

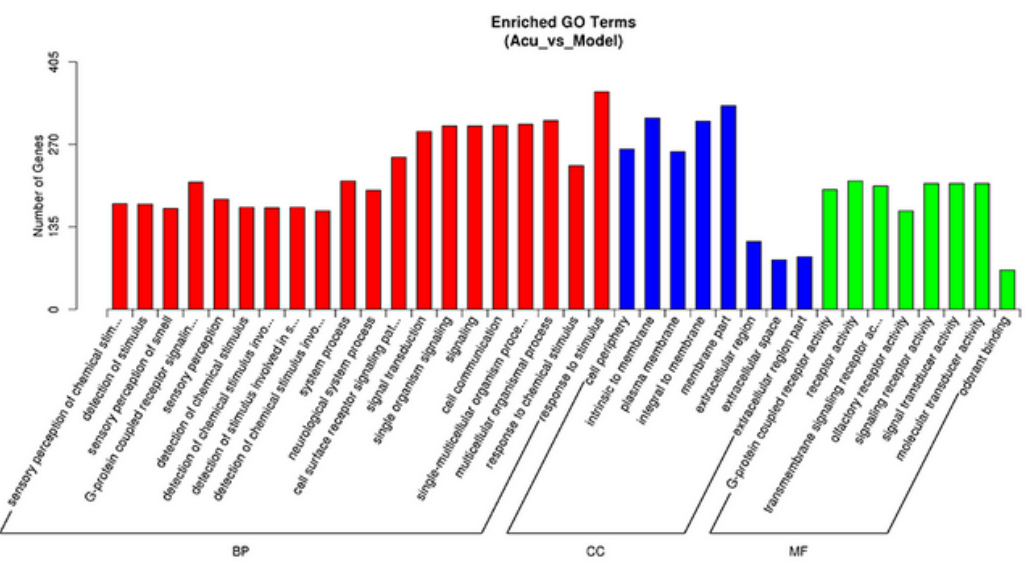

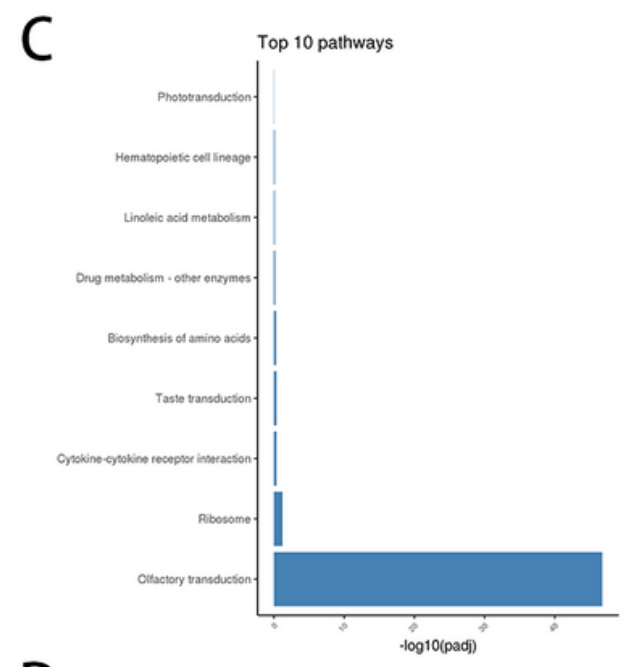

D

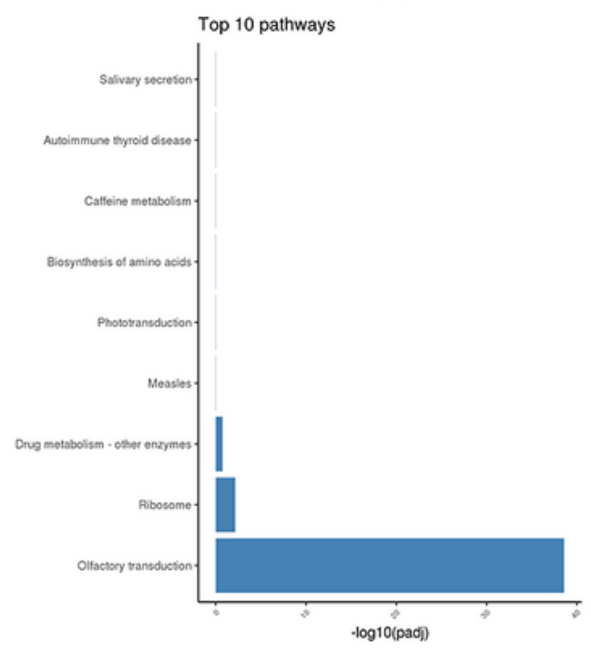

Figure 8

Bioinformatics analysis of the IncRNAs. The most significant GO terms of target mRNAs of IncRNAs that were differentially expressed across domains of biological processes (BP), cellular components (CC), and molecular functions (MF). GO, Gene Ontology; Acu, acupuncture group; Model, model group; Normal, Normal group. The vertical coordinates on the left represent the percent of genes, whereas the one on the right represents the number of genes. (A) The 37 most significant GO terms of target mRNAs of IncRNAs that were differentially expressed between the model and normal groups. (B) The 36 most significant GO terms of target mRNAs of IncRNAs that were differentially expressed between the acupuncture group and model group. (C) The 10 most prominent KEGG pathways of the comparison between acupuncture and model groups. (D) The 10 most prominent KEGG pathways of the comparison between model and acupuncture groups. 
A

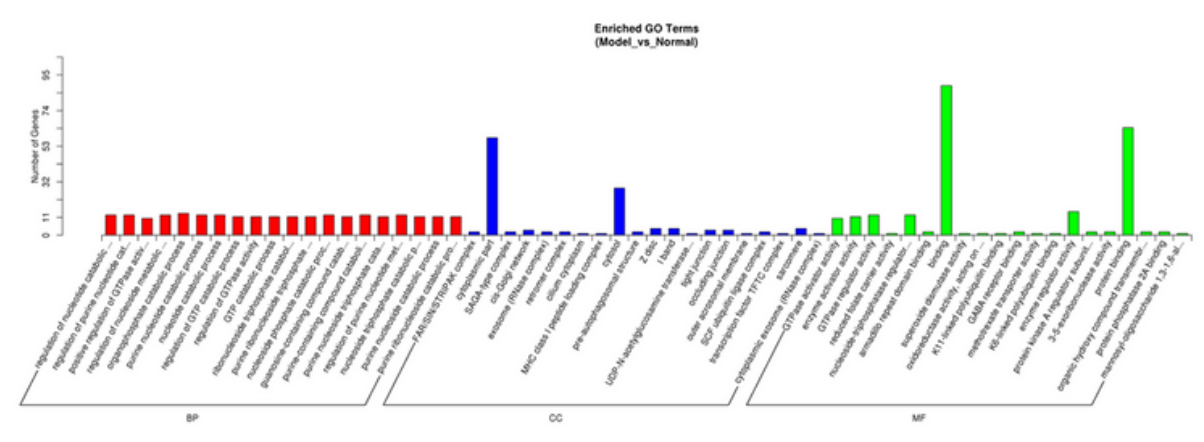

B

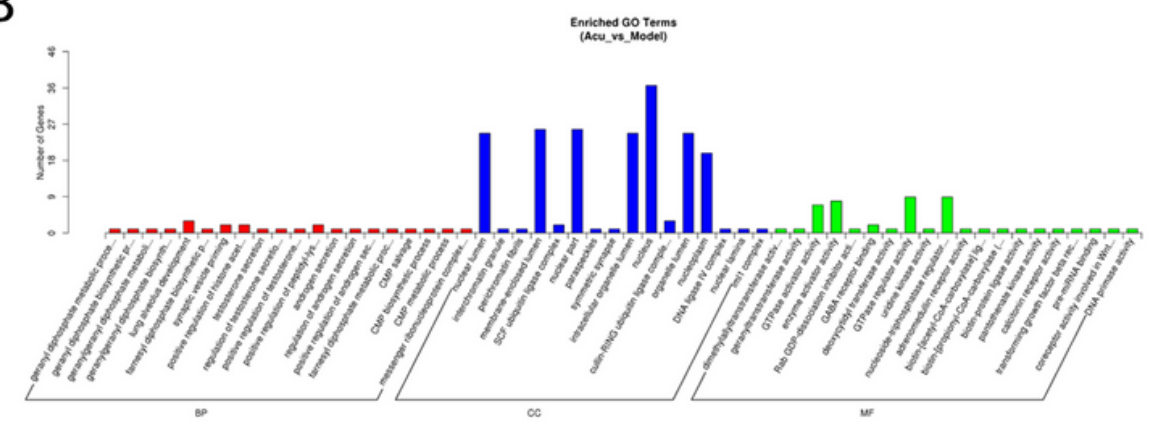

C

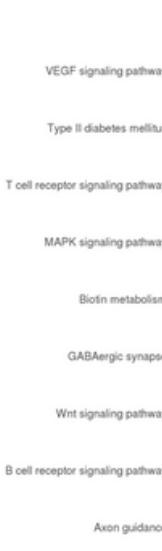

D

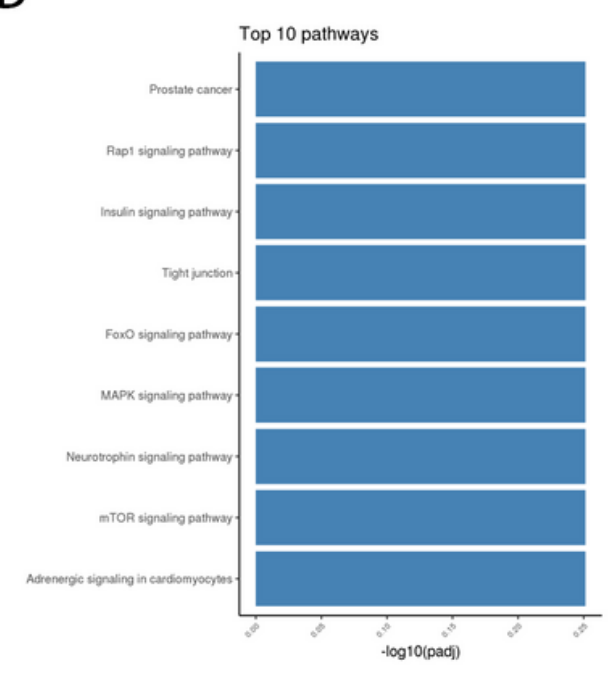

Figure 9

Bioinformatics analysis in the circRNAs. The most significant GO terms for circRNAs that were differentially expressed across the domains of biological processes (BP), cellular components (CC), and molecular functions (MF). GO, Gene Ontology; Acu, acupuncture group; Model, model group; Normal, Normal group. The vertical coordinates on the left represent the percent of genes, whereas the one on the right represents the number of genes. (A) The significant GO terms of circRNAs that were differentially expressed between the model and normal groups. (B) The significant GO terms for circRNAs that were differentially expressed between the acupuncture group and model group. (C) The most prominent KEGG pathways of the comparison between acupuncture and model groups. (D) The most prominent KEGG pathways of the comparison between the model and acupuncture groups. 
A

Acu_vs_Model_miRNA_Inc_mRNA_all

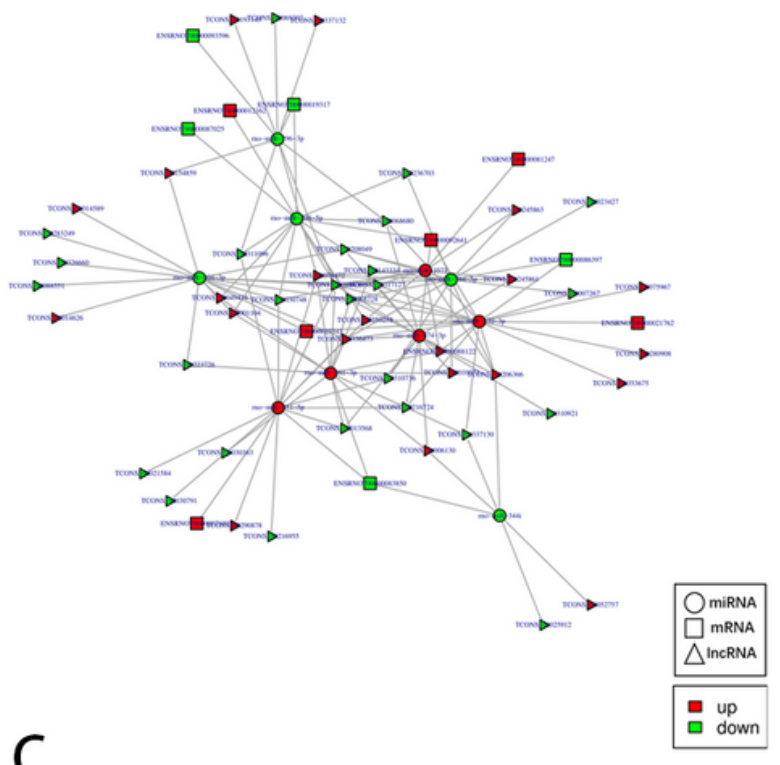

Model_vs_Normal_miRNA_Inc_mRNA_all

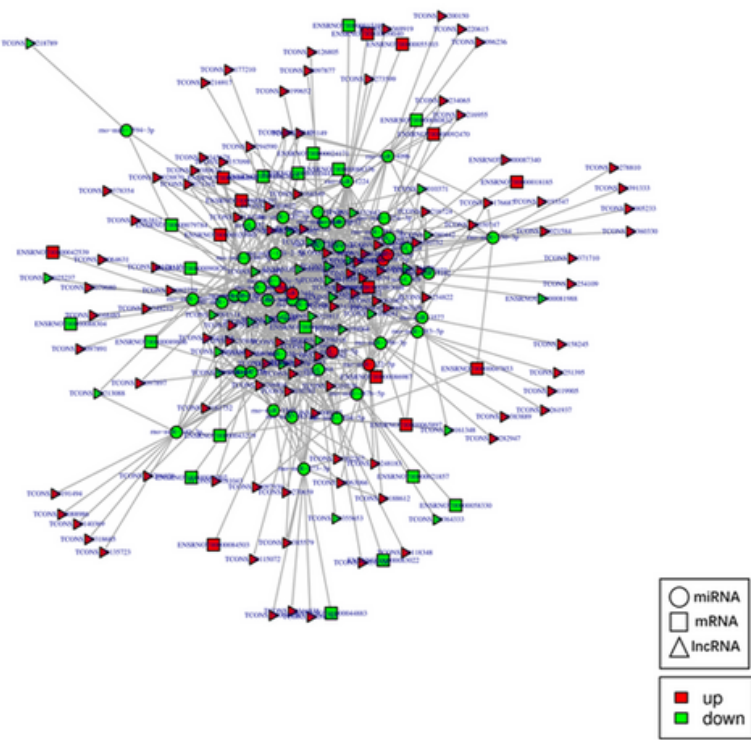

B

Acu_vs_Model_miRNA_circ_mRNA_all

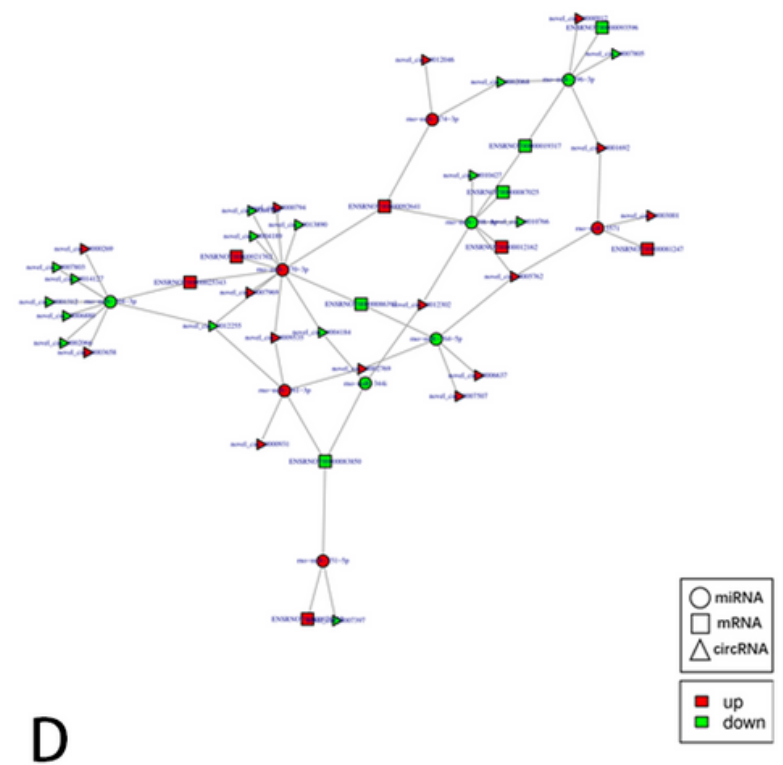

Model_vs_Normal_miRNA_circ_mRNA_all

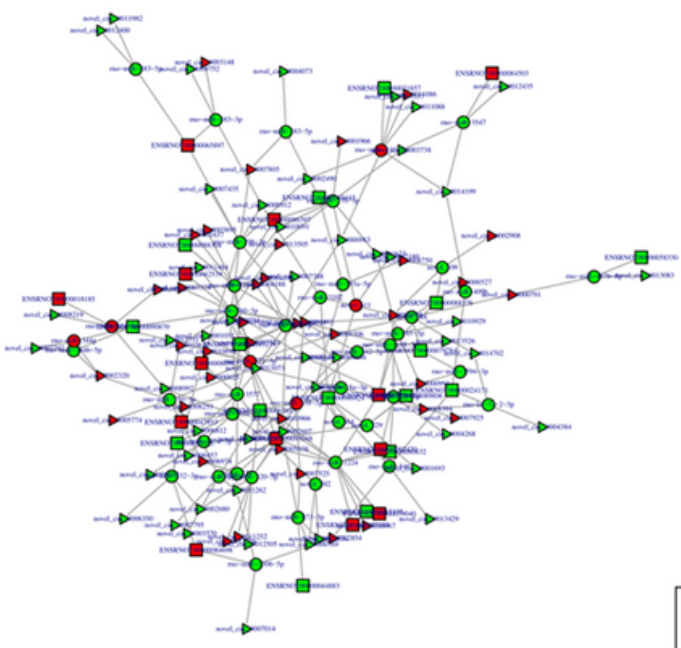

\section{Figure 10}

Bioinformatics analysis in the circRNAs. The most significant GO terms for circRNAs that were differentially expressed across the domains of biological processes (BP), cellular components (CC), and molecular functions (MF). GO, Gene Ontology; Acu, acupuncture group; Model, model group; Normal, Normal group. The vertical coordinates on the left represent the percent of genes, whereas the one on the right represents the number of genes. (A) The significant GO terms of circRNAs that were differentially expressed between the model and normal groups. (B) The significant GO terms for circRNAs that were 
differentially expressed between the acupuncture group and model group. (C) The most prominent KEGG pathways of the comparison between acupuncture and model groups. (D) The most prominent KEGG pathways of the comparison between the model and acupuncture groups.

\section{Supplementary Files}

This is a list of supplementary files associated with this preprint. Click to download.

- TableS1QCstat.xls

- Tables2MapStat.xls

- TableS3annogenes.FPKM.xls

- OnlineDataSupplement.docx 